\title{
Performance Evaluation of Cooperative Relaying for MAC Protocols
}

\author{
Waleed M. Bahgat \\ Department of Information \\ Technology \\ Faculty of Computers and \\ Information \\ Mansoura University, Mansoura, \\ Egypt. \\ Department of Computer Science \\ and Informatics, Taibah University, \\ KSA
}

\author{
Mansoor M. Al-dabaa \\ Department of electronics and \\ communication engineering, \\ Mansoura University, Mansoura, \\ Egypt \\ Ministry of Higher Education and \\ Scientific Research, Yemen
}

\author{
Hassan H. Soliman \\ Professor at faculty of computers \\ and information, Mansoura \\ University, Mansoura, Egypt
}

\begin{abstract}
Recently, Cooperative relaying in wireless networks is a challenging research topic due to its ability to mitigate negative fading in wireless networks. For this reason, this paper evaluates the overall throughput, with and without cooperation, in cooperative relaying wireless network. Hence, four different scenarios are proposed to evaluate the performance of four different cooperative MAC protocols. These protocols are cooperative CSMA, cooperative CSMA/CA, ideal cooperative MAC and cooperative network coding. In addition, this paper drives the mathematical model for these protocols in the proposed scenarios. Afterwards, the overall throughput, with and without cooperative relaying, is evaluated for all scenarios by using the four cooperative MAC protocols. Finally, the paper determines whether the proposed cooperative MAC protocols are beneficial or not in the four scenarios. Moreover, the regions where cooperative relaying protocols are beneficial are discussed for each scenario and the reasons for that. The results show that the cooperative network coding protocol outstands the other protocols.
\end{abstract}

\section{General Terms}

Network management, Network protocols, MAC layer Algorithms.

\section{Keywords}

Networks coding, Cooperative MAC Protocols, Cooperative Network Coding, cooperative CSMA, cooperative CSMA/CA, idea cooperative MAC

\section{INTRODUCTION}

In the last years, cooperative relaying in wireless networks becomes an active area of research since it achieves spatial diversity in a distributed manner by exploiting the broadcast nature of wireless networks. In cooperative relaying, the neighboring nodes, which overhear data packets can help to forward these packets to the specific destination when that is necessary. In this way, multiple copies of the same packet are transmitted to the destination through independent fading channels. This improves system performance in terms of the transmission reliability, capacity, overall throughput and power consumption [1].

Most of the previous researches are focused on the study of the benefits of cooperative relaying in the physical layer. Recently, many of the researches are focused on the impact of and requirement of cooperative relaying in higher layers, such as the medium access control MAC layers. From this perspective, three key issues need to be addressed before design cooperative MAC protocols. First, when to cooperate? The cooperation is used when it's necessary and beneficial; this depends on the channel state between source and destination which vary from time to time. Second, whom to cooperate with? There is a tradeoff between using one relay or multiple relays where choosing one relay (the best of the available relay) give the best performance (low interface, the minimum power used and lower overhead). Whereas using multiple relays improve the diversity, but increase the overhead in the network. Third, how to protect cooperative transmission? The collision may occur during the cooperative transmission, so the MAC protocol must be used to protect transmission by using different collision avoidance techniques [2] [3].

Cooperative relaying can be classified to proactive and reactive scheme by depending on the cooperative strategies. In proactive scheme, the relay node selection takes place before packet transmission; where the relay node listens to the direct transmission and retransmits packets when necessary. While in a reactive scheme, the relay node selection takes place only when a failure occurs in the direct link. [4][5].

There are two methods to forward the received packets at the relay. First, always forward all packets that are received by the relay. This method is applied when destination cannot receive most of the packets via the direct transmitter. Second, the relay node forward only packets that don't receive at the destination. This method is applied when majority of packets are correctly received to the destination via the direct transmitter [6].

In cooperative relaying, the relay node can forward packets according to different strategies, such as Amplify-andForward AF [1], Decode-and-Forward DF [1], composed-andForward CF [7], coded cooperation CC [8], as shown in fig. 1. In $\mathrm{AF}$, the relay node amplifies packets that are received, then forward to the destination. While in the DF, the relay node decode packets that are received, then re-encode and forward to the destination. In this paper, the DF scheme is used since this technique is more practical than other protocols. 

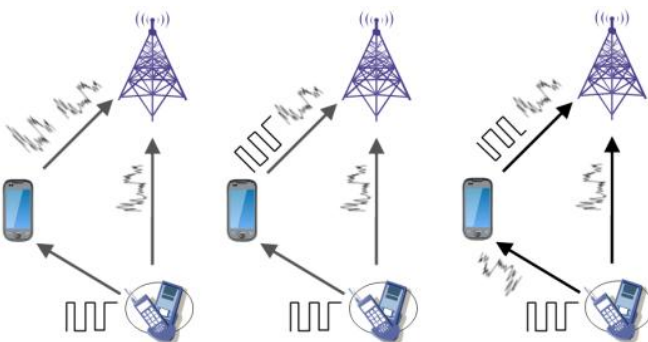

(a). Amplify and Forward $\begin{array}{ll}\text { (b). Decode and Forward (c). Coded cooperation } & \end{array}$

Fig. 1. Cooperative relaying strategies

There are two types of MAC protocols, channel allocationbased MAC and contention-based MAC and. These protocols can be extended for cooperative scenarios, for example, the authors of [9] introduce and classifies the cooperative MAC based on TDMA, and CDMA.

In this paper, we propose four different scenarios by using four different kinds of cooperative MAC protocols. Afterwards, the mathematical model for these protocols is driven in the suggested scenarios. Afterwards, the overall throughput with and without cooperative relaying is evaluated for all scenarios by using the four cooperative MAC protocols. We show whether the cooperative MAC protocols are beneficial or not, for each protocol is identified in the four scenarios. In addition, the regions where cooperative relaying is beneficial for each scenario are determined. Finally, the reasons that make that region are beneficial or not are discussed for the four different scenarios.

The rest of this paper is organized as follows. Frist, the cooperative network coding is explained, in section 2. Then, the related work is presented, in section 3 . In section 4, the analysis of cooperative MAC protocols is driven. The cooperative MAC protocols is evaluated, in section 5. Finally, conclude the paper in section 6 .

\section{COOPERATIVE NETWORK CODING}

Network coding is studied by the authors of [10]. In this technique, the relay nodes mix or encode multiple packets together before forwarding them to the destination. This technique is very helpful to reduce the redundancy and increase the overall throughput in wireless networks. In cooperative relaying, the neighboring nodes that overhearing packets can retransmit packets when necessary, this technique has been employed as an effective technique to deal with the negative channel fading and improve overall throughput in wireless networks. Cooperative network coding technique combines between the advantages of cooperative relaying and network coding since one relay can help more than one node at the same time. On the other hand, it improves the expected number of correctly received, the probability of successful reception, the decoded information system, overall throughput in the network. Moreover, it enhances the system throughput, minimizing energy consumption and minimizing communication delay. This leads to quality improvement in wireless networks.

In traditional cooperative relaying, the relay node behaves in a selfless way in which it is always willing to help other nodes. Such a behavior requires the node to postpone its own queuing packets. Thus, it is not encouraged in a real network.

To enable the node to help other node retransmit while delivering its own data at the same time, Munari et al. Proposed a Hybrid Cooperative Network Coding (HCNC) protocol named Phoenix [11] to take advantage of network coding in cooperative relaying. The key idea that underpins Phoenix is to allow a relay node that retransmits a corrupted packet for another node to encode together a packet of its own.

Fig.2 present the basic idea of cooperative network coding. Nodes $S_{1}$ and $S_{2}$ want to send packet $A$ and $B$ to nodes $D_{1}$ and $\mathrm{D}_{2}$, respectively. When the transmission via the direct link between $S_{1}$ and $D_{1}$ or between $S_{2}$ and $D_{2}$ or both fails, the node $R$ can help $S_{1}$ and $S_{2}$ to retransmit packets $A$ and $B$ simultaneously without affecting each other. Node $R$ combines packets $\mathrm{A}$ and $\mathrm{B}$ together.

\section{RELATED WORK}

Cooperative relaying accomplishes spatial diversity by transmitting multi-copies of the same messages, which enhance the performance of the system. Over recent years, increase research efforts on cooperative relaying especially in MAC layers. These are some of the research in the cooperative MAC protocols. The authors in [12] propose a distributed MAC protocol with automatic repeat request. This protocol enhances the power and throughput in the system. The authors in [13] propose a novel cooperative triple busy tone multiple access to coordinate medium usage and solve an exposed terminal problem. The advantage this protocols can be chosen best relay without disturbing existing transmission. In [14] the authors propose which uses receive beam forming in ad hoc network nodes with smart antennas. Selective CSMA uses carrier sensing only if original packet. These significant capacity improvements are possible. The authors in [15] propose a cooperative CSMA/CA MAC (named CoCHCSMA/ CA MAC) which tailors 802.11 distributed coordination function proposed for $\mathrm{CH}$ based SRMC CRNs. As suggested, COCH-SCMA/CA MAC is suitable for sparse CRNs. in [15] the authors propose a cooperative CSMA/CA MAC (named CoCHCSMA/ CA MAC) which tailors 802.11 distributed coordination function proposed for $\mathrm{CH}$ based SRMC CRNs. As suggested, COCH-SCMA/CA MAC is suitable for sparse CRNs.

Authors of [16] Study the performance of CSMA/CA, cooperative CSMA and ideal cooperative MAC use two simple scenarios. The impact of relaying for different case is studded. They drive the overall network throughput and probability of packet error relation. However, the protocols performance not investigated on complex scenarios.

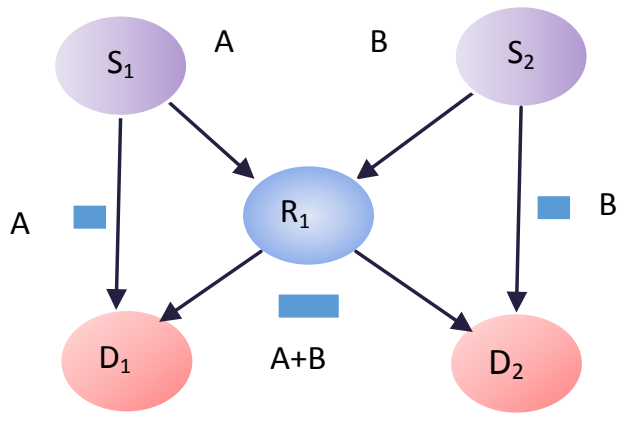

Fig. 2. The basic idea of cooperative network coding

In this paper, we propose four different scenarios and we drive the overall throughput mathematically. The performance with and without cooperative relaying is compared. Then, the regions in which the cooperative relaying is beneficial are determined. Besides, the cooperative CSMA and cooperative CSMA/CA, the cooperative network coding and propose the best network topology is investigated for each MAC protocol. 


\section{ANALYSIS OF COOPERATIVE MAC PROTOCOLS}

In this section, the overall throughput with and without cooperation is driven for four kinds of different scenarios by using four kinds of cooperative MAC protocols (cooperative CSMA protocol, cooperative CSMA/CA protocol, ideal cooperative MAC protocol and cooperative network coding protocol).

The following assumptions are applied in all scenarios with and without cooperation.

- All packets have equal size and priority.

- Size of packets is not specified since it is unnecessary in the analysis.

- Communication channels are divided into time slots according to the packet length.

- When a new data packet arrives at a given time slot $\mathrm{i}$, the probability that this packet is transmitted by node $S_{i}$ is $P_{i}$ where $i=1,2 \ldots n$.

- Queue at each node does not stack packets, which means that if they arrived packet is not sent at the current time slot, it will be dropped.

- The Probability of a packet error between $S_{1}-D_{1}$, $\mathrm{S}_{2}-\mathrm{D}_{2}$ and $\mathrm{S}_{\mathrm{n}}-\mathrm{D}_{\mathrm{n}}$ are denoted by $\propto_{1}, \propto_{2}, \ldots$ and $\propto_{\mathrm{n}}$, respectively.

In addition, the following assumptions are applied when cooperative relaying is employed.

- The DF method is used to forward packets from the relay.

- The relay retransmit packets only when the direct transmission fails.

- The transmission rate is 100 to 500 meters and power are the same for sources, destinations, and relays.

- The error probabilities on links from the sources to the relays are $S_{1}-R, S_{2}-R, \ldots S_{n}-R$ are denoted by $\propto_{\mathrm{S} 1 \mathrm{R}}, \propto_{\mathrm{S} 2 \mathrm{R}}, \ldots$ and $\propto_{\mathrm{SnR}}$. While error probabilities on links from the relay to the destination are dented by, $\mathrm{R}-\mathrm{D}_{1}, \mathrm{R}-\mathrm{D}_{2}, \ldots, \mathrm{R}-\mathrm{D}_{\mathrm{n}}$ and, $\propto_{\mathrm{RD} 1}, \propto_{\mathrm{RD} 2} \ldots, \propto_{\mathrm{RDn}}$, respectively.

- $\quad$ End-to-end packet error probability on path $S_{j}-R-D_{j}$ where $\mathbf{j} \in\{1,2, \ldots, \mathbf{n}\}$ is expressed in ref. [16] as follow:

$\propto_{R j}=1-\left(-\propto_{S j R}\right)\left(1-\propto_{R j D}\right)$

Table.1. Parameters that used in this paper

\begin{tabular}{|c|c|}
\hline Parameters & Explanation of parameters \\
\hline $\mathrm{P}_{1}, \mathrm{P}_{2}, \ldots$, and $\mathrm{P}_{\mathrm{n}}$ & $\begin{array}{l}\text { Transmitted probabilities between } \\
S_{1}, S_{2}, \ldots \text { and } S_{n} \text {, respectively. }\end{array}$ \\
\hline$\propto_{1}, \propto_{2}, \ldots$, and $\propto_{2}$ & $\begin{array}{l}\text { Packet error probability between } S_{1^{-}} \\
D_{1}, S_{2}-D_{2} \ldots \text { and } S_{n}-D_{n} \text {, respectively. }\end{array}$ \\
\hline $\begin{array}{ll}\propto_{\mathrm{S}_{1 \mathrm{R} 1}}, & \propto_{\mathrm{S}_{2 \mathrm{R} 2}, .,, \text { and }} \\
\propto_{\mathrm{S}_{\mathrm{nRn}}} & \end{array}$ & $\begin{array}{l}\text { Packet error probability between } S_{1^{-}} \\
R_{1}-D_{1}, S_{2}-R_{2}-D_{2} \ldots \text { and } S_{n}-R_{n}-D_{n} \\
\text { respectively. }\end{array}$ \\
\hline$\propto_{\mathrm{R} 1}, \propto_{\mathrm{R} 2}$ and $\propto_{\mathrm{n}}$ & $\begin{array}{l}\text { Packet error probability between } S_{1^{-}} \\
R_{1}-D_{1}, S_{2}-R_{2}-D_{2}, \ldots \text { and } S_{n}-R_{n}-D_{n}\end{array}$ \\
\hline
\end{tabular}

\begin{tabular}{|c|l|}
\hline & respectively. \\
\hline $\mathcal{R}_{\text {co }}$ & $\begin{array}{l}\text { Overall throughput with } \\
\text { cooperation. }\end{array}$ \\
\hline $\mathcal{R}$ & $\begin{array}{l}\text { Overall throughput without } \\
\text { cooperation. }\end{array}$ \\
\hline $\mathrm{K}$ & $\begin{array}{l}\text { Total available time slots for } \\
\text { protocol operation. }\end{array}$ \\
\hline $\mathrm{K}$ & $\begin{array}{l}\text { Number of time slots available for } \\
\text { source transmission. }\end{array}$ \\
\hline $\mathrm{q}_{\mathrm{s}}$ & $\begin{array}{l}\text { Ratio time slot available for the } \\
\text { source to the total available time slot } \\
\text { for protocol. }\end{array}$ \\
\hline
\end{tabular}

\subsection{Scenario one of cooperative relaying}

This scenario contains two sources $\left(S_{1}, S_{2}\right)$, two destinations $\left(D_{1}, D_{2}\right)$ and two relays $\left(R_{1}, R_{2}\right)$, as shown in fig. 3. Node $R_{1}$ is out of the range of $D_{2}$ and $R_{2}$ but is in the range of $S_{1}, D_{1}$ and $S_{2}$. So, node $R_{1}$ can retransmit packets between $S_{1}$ and $D_{1}$ only when the direct transmission between them fails and these packets are received correctly from $S_{1}$. While node $R_{2}$ is out of the range $D_{1}, S_{1}$ and $R_{1}$ but in the range of $S_{2}$ and $D_{2}$. Therefore, $R_{2}$ is used as cooperative relaying between $S_{2}$ and $\mathrm{D}_{2}$ only when the direct transmission between them fails and these packets are received correctly from $\mathrm{S}_{2}$. Although the simplicity of this scenario, it provides an important estimation for determining what kinds of cooperative MAC protocols are beneficial when $R_{1}$ and $S_{2}$ are in the same of transmit range of each other, as shown in fig. 3 .

In this scenario, the overall throughput with and without cooperation is driven. Assume, $\mathrm{P}_{1}$ and $\mathrm{P}_{2}$ are transmitted probabilities from $S_{1}$ and $S_{2}$, respectively. While $\propto_{1}$ and $\propto_{2}$ are the packet error probability from $S_{1}-D_{1}$, and $S_{2}-D_{2}$, respectively. In addition, $\propto_{S 1 R}$ and $\propto_{S 2 R}$ are packet error probability from $S_{1}-R_{1}$ and $S_{2}-R_{2}$, respectively. Also, assume $\propto_{R 1}$ and $\propto_{R 2}$ are the packet error probability from $S_{1}-R_{1}-D_{1}$ and $\mathrm{S}_{2}-\mathrm{R}_{2}-\mathrm{D}_{2}$, respectively.

\subsection{Overall throughput without cooperation}

First, the overall throughput when cooperative relaying is not applied is derived. All MAC protocols that are studied here provide the same throughput when singing overhead is ignored. The overall throughput without cooperation in this scenario is calculated as in ref [17]:

$$
\mathcal{R}=\mathrm{P}_{1}\left(1-\propto_{1}\right)+\mathrm{P}_{2}\left(1-\propto_{2}\right)
$$
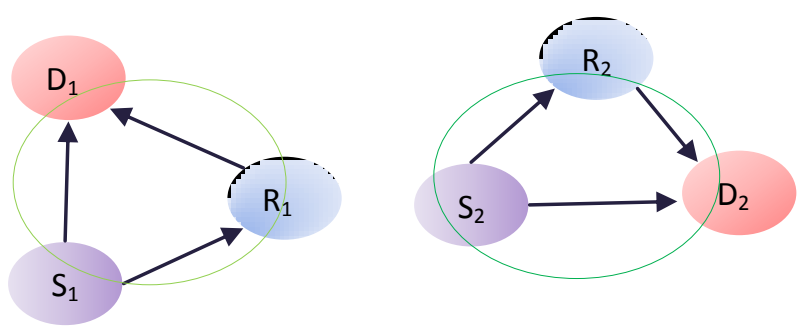

Fig. 3. Scenario one of cooperative relaying 


\subsection{Overall throughput with cooperation}

The overall throughput when cooperative relaying is applied for three different cooperative MAC protocols is driven as follow:

\subsubsection{Cooperative CSMA/CA protocol}

When node $S_{1}$ transmit, node $S_{2}$ cannot transmit due to $S_{1}$ transmission Request-To-Send RTS and Clear-To-Send CTS handshake before transmitting packets of distributed coordinated function DCF and vice versa. Therefore, Transmission at the same time by $R_{1}$ and $S_{2}$ is impossible, although, $S_{2}$ transmit to $D_{2}$, while $R_{2}$ retransmitted to $D_{1}$. This problem occurs due to lack of synchronization between $S_{2}$ and $\mathrm{R}_{1}$ namely an expose terminal problem. Node $\mathrm{R}_{1}$ or $\mathrm{R}_{2}$ retransmit packets when direct link between $S_{1}$ and $D_{1}$ or between $S_{2}$ and $D_{2}$ failure, respectively. When nodes $R_{1}$ or $R_{2}$ retransmit packets, nodes $S_{1}$ or $S_{2}$ cannot transmit any packets (the slot time in $S_{1}$ or $S_{2}$ is not available) until $D_{1}$ or $D_{2}$ correctly received packet or the transmission time is out in $\mathrm{R}_{1}$ or $\mathrm{R}_{2}$, respectively. $\mathrm{K}$ is calculated in as:

$$
\begin{gathered}
\mathrm{K}=\mathrm{K}\left[1-\left(0.5 \mathrm{P}_{1} \mathrm{P}_{2}+\mathrm{P}_{1}\left(1-\mathrm{P}_{2}\right)\right) \propto_{1}\left(1-\propto_{\mathrm{S} 1 \mathrm{R} 1}\right)\right. \\
\left.-\left(0.5 \mathrm{P}_{1} \mathrm{P}_{2}+\mathrm{P}_{2}\left(1-\mathrm{P}_{1}\right)\right) \propto_{2}\left(1-\propto_{\mathrm{S} 2 \mathrm{R} 2}\right)\right]
\end{gathered}
$$

Where $\mathrm{q}_{\mathrm{S}}=\hat{\mathrm{K}} / \mathrm{K}$

The overall throughput in this protocol is calculated, as follow:

$$
\begin{aligned}
& \mathcal{R}_{\mathrm{CO}}=\mathrm{q}_{\mathrm{S}}\left[( 0 . 5 \mathrm { P } _ { 1 } \mathrm { P } _ { 2 } + \mathrm { P } _ { 1 } ( 1 - \mathrm { P } _ { 2 } ) ) \left(\left(1-\propto_{1}\right)+\propto_{1}\left(1-\propto_{\mathrm{R} 1}\right)\right.\right. \\
& )+\left(0.5 \mathrm{P}_{1} \mathrm{P}_{2}+\mathrm{P}_{2}\left(1-\mathrm{P}_{1}\right)\right)\left(\left(1-\propto_{2}\right)+\propto_{2}\left(1-\propto_{\mathrm{R} 2}\right)\right) \\
& +0.5 \mathrm{P}_{1} \mathrm{P}_{2}\left(1-\propto_{1}\right)
\end{aligned}
$$

The first row in equation (4) corresponds to the packets that transmit when $S_{1}$ start transmitting first; during this period $S_{2}$ cannot transmit until $S_{1}$ finish transmits or time out. While the second and three rows corresponds to the packets that transmit when $\mathrm{S}_{2}$ start transmitting first.

\subsubsection{Ideal cooperative MAC protocol}

In this protocol, $S_{2}$ and $R_{1}$ can transmit packets simultaneously without impact of each other since the exposed terminal problem is solved. Therefore, the overall throughput improvement in both links $S_{1}-R_{1}-D_{1}$ and $S_{1}-R_{1}-D_{1}$ without impact of each other. $\mathrm{q}_{\mathrm{s}}$ is calculated as:

$$
\begin{aligned}
& \mathrm{q}_{\mathrm{S} 1}=1-\mathrm{P}_{1} \propto_{1}\left(1-\propto_{\mathrm{S} 1 \mathrm{R} 1}\right) \\
& \mathrm{q}_{\mathrm{S} 2}=1-\mathrm{P}_{2} \propto_{2}\left(1-\propto_{\mathrm{S} 2 \mathrm{R} 2}\right)
\end{aligned}
$$

- $\quad \mathrm{q}_{\mathrm{s} 1}$ and $\mathrm{q}_{\mathrm{s} 2}$ represent the ratio of time slot that is available for $S_{1}$ and $S_{2}$ to the total available time slot for protocol operation between $S_{1}-R_{1^{-}}$ $D_{1}$ and $S_{2}-R_{2}-D_{2}$, respectively.

The overall throughput in this protocol is calculated as:

$$
\begin{aligned}
\mathcal{R}_{\mathrm{CO}} & =\mathrm{q}_{\mathrm{S} 1} \mathrm{P}_{1}\left(\left(1-\propto_{1}\right)+\propto_{1}\left(1-\propto_{\mathrm{R} 1}\right)\right)+\mathrm{q}_{\mathrm{S} 2} \mathrm{P}_{2}\left(\left(1-\propto_{2}\right)\right. \\
& \left.+\propto_{2}\left(1-\propto_{\mathrm{R} 2}\right)\right)
\end{aligned}
$$

\subsubsection{Cooperative network coding protocol}

In this protocol, $S_{2}$ and $R_{1}$ can transmit packets simultaneously without impact of each other. Assume the time slot in all nodes is always available, which means that all sources and relays can transmit packets simultaneously without affect each other. The overall throughput in this protocol is calculated as:

$$
\begin{aligned}
& \mathcal{R}_{\mathrm{CO}}=\mathrm{P}_{1}\left(\left(1-\propto_{1}\right)+\propto_{1}\left(1-\propto_{\mathrm{R} 1}\right)\right)+\mathrm{P}_{2}\left(\left(1-\propto_{2}\right)\right. \\
& \left.+\propto_{2}\left(1-\propto_{\mathrm{R} 2}\right)\right)
\end{aligned}
$$

\subsection{Scenario two of cooperative relaying}

This scenario conations two sources $\left(\mathrm{S}_{1}, \mathrm{~S}_{2}\right)$, two destinations $\left(D_{1}, D_{2}\right)$ and one relay $(R)$. Node $R$ is in the range of transmitting $S_{1}$ and $S_{2}$, as shown in fig. 4. All pairs can transmit packets without affecting each other when cooperative relaying is not employed. This scenario is studied to evaluate what kinds of cooperative MAC protocols are beneficial when $\mathrm{R}$ is in the transmit range of all the couples, as shown in fig. 4 . In this scenario, assume $\mathrm{P}_{1}$ and $\mathrm{P}_{2}$ are the probability that packets are transmitted from $S_{1}$ and $S_{2}$, respectively. In addition, $\propto_{1}$ and $\propto_{2}$ represent the packet error probability from $S_{1}-D_{1}$, and $S_{2}-D_{2}$, respectively. Also, $\propto_{S 1_{R}}$ and $\propto_{S 2_{R}}$ are the probability of a packet error from $S_{1}-R_{1}$ and $\mathrm{S}_{2}-\mathrm{R}_{2}$, respectively. In addition, $\propto_{\mathrm{R} 1}$ and $\propto_{R 2}$ are the probability of packet error from $S_{1}-R_{1}-D_{1}$ and $S_{2}-R-D_{2}$, respectively.

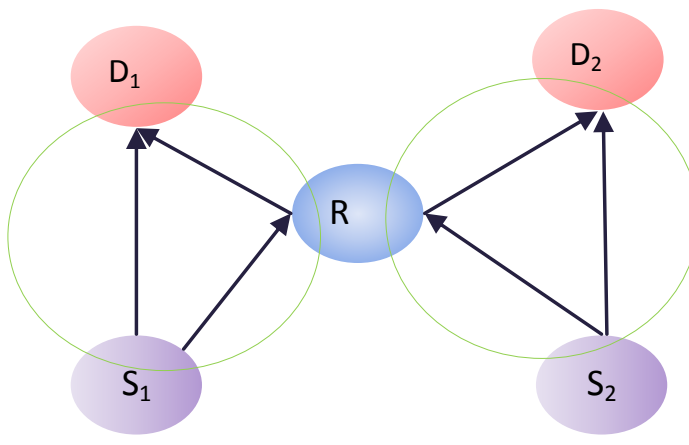

Fig. 4. Scenario two of cooperative relaying

\subsection{Overall throughput without cooperation}

First, we drive the overall throughput when cooperative MAC protocols are not used and a singling overhead is ignored. In this case, all MAC protocols provide the same overall throughput. The overall throughput, in this case, is calculated as in ref [16]:

$\mathcal{R}=\mathrm{P}_{1}\left(1-\propto_{1}\right)+\mathrm{P}_{2}\left(1-\propto_{2}\right)$

\subsection{Overall throughput with cooperation}

Second, the overall throughput with cooperation is driven when three different cooperative MAC protocols are used, as follows:

\subsubsection{Cooperative CSMA protocol}

In this protocol, when $\mathrm{S}_{1}$ and $\mathrm{S}_{2}$ transmit packets simultaneously (when $\mathrm{P}_{1}=\mathrm{P}_{2}=1$ ), node $\mathrm{R}$ not work due interference. In this case, the overall throughput with and without cooperation is equal. Node $R$ can receive and retransmit packets when the direct link between $S_{1}$ and $D_{1}$ or $S_{2}$ and $D_{2}$ failure. When node $R$ transmit packets, $S_{1}$ and $S_{2}$ cannot transmit packets until $\mathrm{R}$ ends of the retransmission or time out. In this protocols, $\mathrm{q}_{\mathrm{s}}$ is calculate as in ref [16]:

$$
\begin{aligned}
& q_{\mathrm{S}}=\left[1-\mathrm{P}_{1}\left(1-\mathrm{P}_{2}\right) \propto_{1}\left(1-\propto_{\mathrm{S} 1 \mathrm{R}}\right)-\mathrm{P}_{2}\left(1-\mathrm{P}_{1}\right)\right. \\
& \propto_{2}\left(1-\propto_{\mathrm{S} 2 \mathrm{R}}\right)
\end{aligned}
$$

The overall throughput in this scenario is calculated as a ratio of packets that successfully received at $D_{1}$ and $D_{2}$ as in ref [16]:

$$
\mathcal{R}_{\mathrm{CO}}=q_{\mathrm{S}}\left[\mathrm{P}_{1}\left(1-\propto_{1}\right)+\mathrm{P}_{2}\left(1-\propto_{1}\right)+\mathrm{P}_{1}\left(1-\mathrm{P}_{2}\right)\right.
$$




$$
\left.\propto_{1}\left(1-\propto_{\mathrm{SR} 1}\right)+\mathrm{P}_{2}\left(1-\mathrm{P}_{1}\right) \propto_{2}\left(1-\propto_{\mathrm{SR} 2}\right)\right]
$$

First and second terms in equation (11) correspond to the possibility of receiving packets through direct link. While the summation of third and fourth terms corresponds to the possibility of receiving packets via $\mathrm{R}$, which is done when all the following points are true.

- The direct transmission fails.

- Other sources does not transmit any packets at the same time.

- Node $\mathrm{R}$ has correctly receives a data packet from $S_{1}$ or $S_{2}$.

- Two-hop path (S-R and R-D) over the cooperative $\mathrm{R}$ is good.

\subsubsection{Cooperative CSMA/CA protocol}

In this protocol, the nodes $S_{1}$ and $S_{2}$ transmits RTS-CTS handshake before transmitting data packets to reserve the channel. Therefore the transmission at the same time by $S_{1}$ and $S_{2}$ are impossible. When $S_{1}$ and $S_{2}$ want to transmit the packet at the same time, each of them has the same probability to win. $\mathrm{q}_{\mathrm{s}}$ is calculated as in ref [16]:

$$
\begin{aligned}
& q_{\mathrm{S}}=\left[1-\left(0.5 \mathrm{P}_{1} \mathrm{P}_{2}+\mathrm{P}_{1}\left(1-\mathrm{P}_{2}\right)\right) \propto_{1}\left(1-\propto_{\mathrm{S} 1 \mathrm{R}}\right)\right. \\
& \left.-\left(0.5 \mathrm{P}_{1} \mathrm{P}_{2}+\mathrm{P}_{2}\left(1-\mathrm{P}_{1}\right)\right) \propto_{2}\left(1-\propto_{\mathrm{S} 2 \mathrm{R}}\right)\right]
\end{aligned}
$$

The overall throughput in this protocol is calculated as in ref [16]:

$\mathcal{R}_{\mathrm{CO}}=q_{\mathrm{S}}\left[\left(0.5 \mathrm{P}_{1} \mathrm{P}_{2}+\mathrm{P}_{1}\left(1-\mathrm{P}_{2}\right)\right)\left(\left(1-\propto_{1}\right)+\propto_{1}\left(1-\propto_{\mathrm{R} 1}\right)\right.\right.$

)$\left.-\left(0.5 \mathrm{P}_{1} \mathrm{P}_{2}+\mathrm{P}_{2}\left(1-\mathrm{P}_{1}\right)\right)\left(\left(1-\propto_{2}\right)+\propto_{2}\left(1-\propto_{\mathrm{R} 2}\right)\right)\right](13)$

\subsubsection{Cooperative Network Coding protocol}

In this protocol, one relay can help more than one node at the same time. Also, we assume that the time slot is available in every time, which means that all sources and relay can transmit data at the same time. The $\mathcal{R}_{\text {co }}$ in this protocol is driven as follow:

$$
\begin{aligned}
& \mathcal{R}_{\mathrm{CO}}=\mathrm{P}_{1}\left(\left(1-\propto_{1}\right)+\propto_{1}\left(1-\propto_{\mathrm{R} 1}\right)\right)+\mathrm{P}_{1}\left(\left(1-\propto_{1}\right)\right. \\
& \left.+\propto_{2}\left(1-\propto_{\mathrm{R} 2}\right)\right)
\end{aligned}
$$

\subsection{Scenario three of cooperative relaying}

This scenario contains three sources $\left(S_{1}, S_{2}\right.$, and $\left.S_{3}\right)$, three destinations $\left(D_{1}, D_{2}\right.$, and $\left.D_{3}\right)$, and one relay $(R)$. Node $R$ is in transmission range of all pairs, but each pair is out of transmission range of all each other, as shown in fig. 5. This scenario is studied to determine what kinds of cooperative MAC protocols are beneficial when one relay help three pairs, as shown in fig. 5. Here, Assume $\mathrm{P}_{1}, \mathrm{P}_{2}$, and $\mathrm{P}_{3}$ are the probability that packets transmitting from $S_{1}, S_{2}$ and $S_{3}$, respectively. In addition, $\propto_{1}, \propto_{2}$ and $\propto_{3}$ are the probability of a packet error between $S_{1}-D_{1}, S_{2}-D_{2}$ and $S_{3}-D_{3}$, respectively. Also, assume $\propto_{S 1_{R}}, \propto_{S 2_{R}}$ and $\propto_{S 3_{R}}$ are the probability of a packet error from $S_{1}-R_{1}, S_{2}-R_{1}$, and $S_{3}-R_{1}$ respectively. Also, $\propto_{R 1}, \propto_{R 2}$ and $\propto_{R 3}$ are the probability of a packet error from $S_{1^{-}}$ $\mathrm{R}-\mathrm{D}_{1}, \mathrm{~S}_{1}-\mathrm{R}-\mathrm{D}_{2}$, and $\mathrm{S}_{3}-\mathrm{R}-\mathrm{D}_{3}$ respectively.

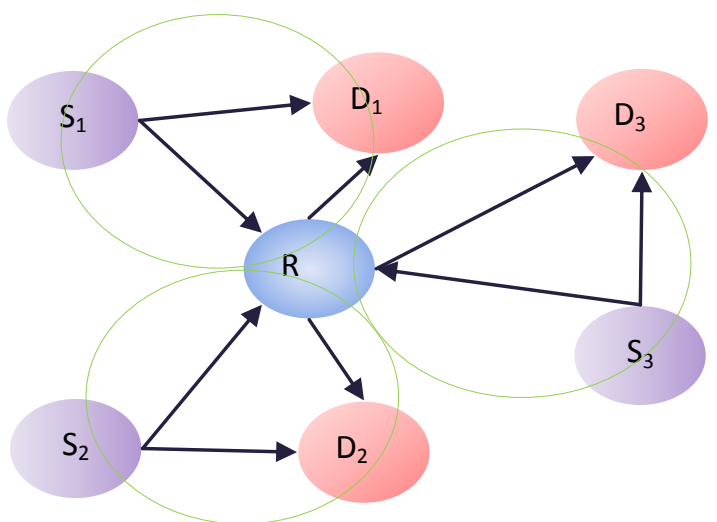

Fig. 5. Scenario three of cooperative relaying

\subsection{Overall throughput without cooperation}

The overall throughput is driven when the cooperative MAC protocol is not employed. The overall throughput without cooperative relaying is calculated as:

$$
\mathcal{R}=\mathrm{P}_{1}\left(1-\propto_{1}\right)+\mathrm{P}_{2}\left(1-\propto_{2}\right)+\mathrm{P}_{3}\left(1-\propto_{3}\right)
$$

\subsection{Overall throughput with cooperation}

The overall throughput is driven when cooperative MAC protocols are applied. The overall throughput drive for three kinds of cooperative MAC protocols as follows:

\subsubsection{Cooperative CSMA protocol}

When Cooperative CSMA applies in this scenario. $\mathrm{q}_{\mathrm{s}}$ is given by:

$$
\begin{aligned}
q_{\mathrm{S}}= & {\left[1-\mathrm{P}_{1}\left(1-\mathrm{P}_{2}\right)\left(1-\mathrm{P}_{3}\right) \propto_{1}\left(1-\propto_{\mathrm{S} 1 \mathrm{R}}\right)\right.} \\
& -\mathrm{P}_{2}\left(1-\mathrm{P}_{1}\right)\left(1-\mathrm{P}_{3}\right) \propto_{2}\left(1-\propto_{\mathrm{S} 2 \mathrm{R}}\right) \\
- & \mathrm{P}_{3}\left(1-\mathrm{P}_{1}\right)\left(1-\mathrm{P}_{2}\right) \propto_{3}\left(1-\propto_{\mathrm{S} 3 \mathrm{R}}\right)
\end{aligned}
$$

The overall throughput in this protocol is calculated as:

$$
\begin{aligned}
\mathcal{R}_{\mathrm{CO}}= & q_{\mathrm{S}}\left[\left(\mathrm{P}_{1}\left(1-\propto_{1}\right)+\mathrm{P}_{2}\left(1-\propto_{2}\right)+\mathrm{P}_{3}\left(1-\propto_{3}\right)\right.\right. \\
& +\mathrm{P}_{1}\left(1-\mathrm{P}_{2}\right)\left(1-\mathrm{P}_{3}\right) \propto_{1}\left(1-\propto_{\mathrm{R} 1}\right) \\
& +\mathrm{P}_{2}\left(1-\mathrm{P}_{1}\right)\left(1-\mathrm{P}_{3}\right) \propto_{2}\left(1-\propto_{\mathrm{R} 2}\right) \\
+\mathrm{P}_{3} & \left.\left(1-\mathrm{P}_{1}\right)\left(1-\mathrm{P}_{2}\right) \propto_{3}\left(1-\propto_{\mathrm{R} 3}\right)\right]
\end{aligned}
$$

First row of the equation (16) representation of the probability that packets are successfully delivered through the direct link. While second, third and fourth rows of equation (16) representation of the probability that packets are successfully delivered through the relay when direct transmission fails.

\subsubsection{Cooperative CSMA/CA protocol}

For this protocol, $\mathrm{q}_{\mathrm{s}}$ is given by:

$$
\begin{gathered}
q_{\mathrm{S}}=\left[1-\left(\left(\frac{1}{3}\right) \mathrm{P}_{1} \mathrm{P}_{2} \mathrm{P}_{3}+\mathrm{P}_{1}\left(1-\mathrm{P}_{2}\right)\left(1-\mathrm{P}_{3}\right)\right)\right. \\
\propto_{1}\left(1-\propto_{\mathrm{S} 1 \mathrm{R}}\right)-\left(\left(\frac{1}{3}\right) \mathrm{P}_{1} \mathrm{P}_{2} \mathrm{P}_{3}+\mathrm{P}_{2}\left(1-\mathrm{P}_{1}\right)\left(1-\mathrm{P}_{3}\right)\right) \\
\propto_{2}\left(1-\propto_{\mathrm{S} 2 \mathrm{R}}\right)-\left(\left(\frac{1}{3}\right) \mathrm{P}_{1} \mathrm{P}_{2} \mathrm{P}_{3}+\mathrm{P}_{2}\left(1-\mathrm{P}_{1}\right)\left(1-\mathrm{P}_{3}\right)\right) \\
\left.\propto_{3}\left(1-\propto_{\mathrm{S} 3 \mathrm{R}}\right)\right]
\end{gathered}
$$

The overall throughput in this protocol is calculated as: 


$$
\begin{aligned}
\mathcal{R}_{\text {co }} & =q_{\mathrm{S}}\left[\left(\left(\frac{1}{3}\right) \mathrm{P}_{1} \mathrm{P}_{2} \mathrm{P}_{3}+\mathrm{P}_{1}\left(1-\mathrm{P}_{2}\right)\left(1-\mathrm{P}_{3}\right)\right)\right. \\
& \left(\left(1-\propto_{1}\right)+\propto_{1}\left(1-\propto_{\mathrm{SR} 1}\right)\right)+\left(\left(\frac{1}{3}\right) \mathrm{P}_{1} \mathrm{P}_{2} \mathrm{P}_{3}\right. \\
& \left.\mathrm{P}_{2}\left(1-\mathrm{P}_{1}\right)\left(1-\mathrm{P}_{3}\right)\right)+\left(\left(1-\propto_{2}\right)+\propto_{2}\left(1-\propto_{\mathrm{SR} 2}\right)\right) \\
& \left(\left(\frac{1}{3}\right) \mathrm{P}_{1} \mathrm{P}_{2} \mathrm{P}_{3}+\mathrm{P}_{3}\left(1-\mathrm{P}_{1}\right)\left(1-\mathrm{P}_{2}\right)\right) \\
& \left(\left(1-\propto_{3}\right)+\propto_{3}\left(1-\propto_{\mathrm{SR} 3}\right)\right)
\end{aligned}
$$

\subsubsection{Cooperative Network Coding protocol} In this protocol, the overall throughput is given by:

$$
\begin{gathered}
\mathcal{R}_{\text {co }}=\mathrm{P}_{1}\left(\left(1-\propto_{1}\right)+\propto_{1}\left(1-\propto_{\mathrm{R} 1}\right)\right) \\
+\mathrm{P}_{2}\left(\left(1-\propto_{2}\right)+\propto_{2}\left(1-\propto_{\mathrm{R} 2}\right)\right) \\
+\mathrm{P}_{3}\left(\left(1-\propto_{3}\right)+\propto_{3}\left(1-\propto_{\mathrm{R} 3}\right)\right)
\end{gathered}
$$

\subsection{Scenario four of cooperative relaying}

According to the results in the previous scenarios, the cooperation in case of cooperative CSMA and cooperative CSMA/CA are non-beneficial in most regions for scenario two and become always non- beneficial in scenario three. The reason for that there is one relay is used for all nodes in scenario two and three. Therefore, we propose using one relay for each transmitting-receiving pair as shown in fig. 6a. Every pair communicates without affecting the other pairs. Then, the equations are generalized in cooperative CSMA, cooperative CSMA/CA and ideal cooperative MAC of overall throughput with and without cooperation.

In fig. $6 b$, one relay is used for all nodes where all nodes including one relay have the ability to transmit and receive packets simultaneously and use cooperative network coding; this increases the throughput and reduces the usage of spectrum and the nodes can be spatial overlap. Hence, the generalized equation of overall throughput with and without cooperation is driven.

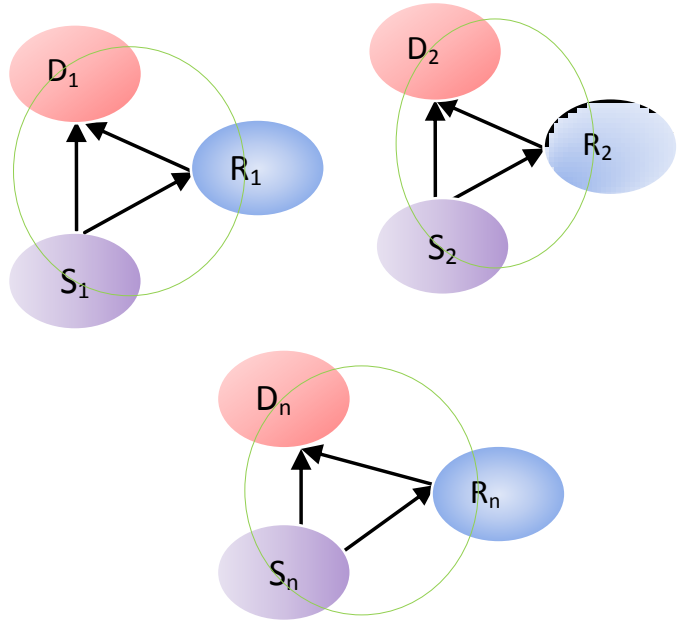

Fig. 6a. Scenario four for cooperative relaying

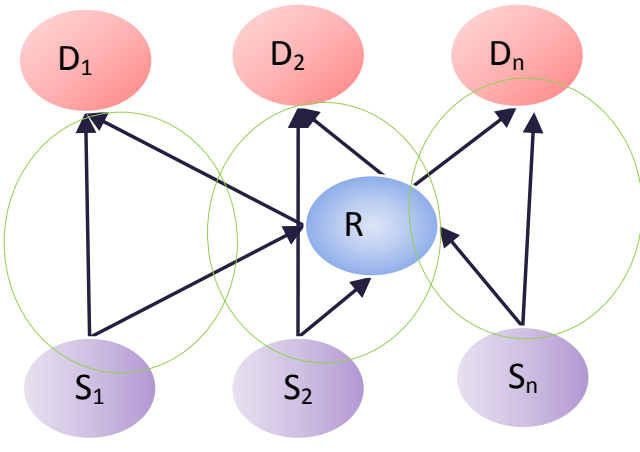

Fig. 6b. Scenario four for cooperative relaying

\subsection{Overall throughput without cooperation}

The overall throughput without cooperation is driven. The same overall throughput is getting in all MAC protocols that are studied here when cooperative relaying is not used. The Overall throughput is calculated by the general equation as follows:

$\mathcal{R}=\sum_{m=1}^{m=n} \mathrm{P}_{m}\left(1-\propto_{m}\right)$

Where $n$ represents the number of sources.

\subsection{Overall throughput with cooperation}

The overall throughput with cooperation is driven. The same overall throughput is got when the following MAC protocols are used, cooperative CSMA, cooperative CSMA/CA and Ideal cooperative MAC. $\mathrm{q}_{\mathrm{s}}$ is given by:

$$
q_{S}=1-\left[\sum_{m=1}^{m=n} \mathrm{P}_{m} \propto_{m}\left(1-\propto_{S m R}\right)\right]
$$

The overall throughput in this case is expressed as:

$$
\mathcal{R}_{C O}=q_{S}\left[\sum_{m=1}^{m=n} \mathrm{P}_{m}\left(\left(1-\propto_{m}\right)+\propto_{m}\left(1-\propto_{R m}\right)\right)\right]
$$

When cooperative network coding is applied as shown in fig. $15 \mathrm{~b}$, the overall throughput is calculated as:

$$
\mathcal{R}_{C O}=\sum_{m=1}^{m=n} \mathrm{P}_{m}\left(\left(1-\propto_{m}\right)+\propto_{m}\left(1-\propto_{R m}\right)\right)
$$

Where $\mathbf{n}$ represents the number of sources.

\section{PERFORMANCE EVALUATION OF COOPERATIVE MAC PROTOCOLS}

In this section, we evaluate the performance of cooperative CSMA/CA protocol, ideal cooperative MAC protocol and cooperative network coding protocol for four different scenarios. The evolution is accomplished in two stages for each scenario.

First, plot ratio overall throughput with cooperation to that without cooperation $\mathcal{R}_{\mathrm{co}} / \mathcal{R}$ versus transmission probability $\mathrm{P}_{1}$ with different value of $\mathrm{P}_{2}=0.2,0.4,0.6$ and 0.8 . Assume the packet error probability between source(s) and destination(s) are $\propto_{1}=\alpha_{2}=\ldots=\propto_{n}=0.3$. In additional, assume the error probability between source(s), destination(s) and relay(s) are zero (optimum relaying) $\propto_{R 1}=\propto_{R 2}=\ldots .=\propto_{R n}=0$.

Second, plot ratio of overall throughput with cooperation to that without cooperation $\mathcal{R}_{\mathrm{co}} / \mathcal{R}$ versus error packet probability $\propto_{1}$ with different value of $\propto_{2}=0.2,0.4,0.6$ and 0.8 . Also, 
assume the fixed values of transmission probability for $\mathrm{S}_{1}$, $\mathrm{S}_{2}, \ldots, \mathrm{S}_{\mathrm{n}}$ are $\mathrm{P}_{1}=\mathrm{P}_{2}=\ldots .=\mathrm{P}_{\mathrm{n}}=0.75$. In additional, assume the errors probability between source(s), destination(s) and relay(s) are zero (optimal relaying) $\propto_{R 1}=\propto_{R 2}=\ldots .=\propto_{R n}=0$.

\subsection{Evaluation of cooperative MAC protocols for scenario one}

The first stage, the ratio $\mathcal{R}_{\text {co }} / \mathcal{R}$ versus $\mathrm{P}_{1}$ with the different value of $\mathrm{P}_{2}=0.2,0.4,0.6$ and 0.8 is plotted. Also, assume $=\propto_{2}$ $=0.3 ; \propto_{1} \propto_{R 1}=\propto_{R 2}=0$. In fig.7a, b, c and d.

Fig.7a shows ratio $\mathcal{R}_{\text {co }} / \mathcal{R}$ versus $\mathrm{P}_{1}$ for cooperative CSMS/CA protocol. The results show the following:

- The value of $\mathcal{R}_{\text {co }} / \mathcal{R}$ decreases when the value of $\mathrm{P}_{2}$ and $\mathrm{P}_{1}$ increases since $\mathrm{R}_{1}$ cannot transmit packets when $\mathrm{S}_{2}$ start transmit packets and vice versa because of the exposed terminal problem between $\mathrm{S}_{2}$ and $\mathrm{R}_{1}$.

- The value of $\mathcal{R}_{\mathrm{co}} / \mathcal{R}$ is less than one in the most regions since $S_{2}$ and $R_{1}$ cannot transmit packets at the same time. For this reason, cooperative CSMA/CA protocol has a negative impact on the overall throughput in this scenario, especially when increasing the value of $\mathrm{P}_{1}$ and $\mathrm{P}_{2}$.

- The value of $\mathcal{R}_{\mathrm{co}} / \mathcal{R}$ decreases constantly when the value of $\mathrm{P}_{1}$ and $\mathrm{P}_{2}$ increase due to increasing the interference between $S_{2}$ and $R_{1}$.

Fig.7b Shows ratio of $\mathcal{R}_{\text {co }} / \mathcal{R}$ versus $\mathrm{P}_{1}$ for ideal cooperative MAC protocol. The results show the following:

- The value of $\mathcal{R}_{\text {co }} / \mathcal{R}$ decreases when the value of $\mathrm{P}_{2}$ increase since the error probability increases as the number of transmitted packets increases.

- The value of $\mathcal{R}_{\text {co }} / \mathcal{R}$ is always greater than one since nodes $R_{1}$ and $S_{2}$ can transmit packets at the same time since an exposed terminal problem is solved for this protocol. For this reason, ideal cooperative MAC is always beneficial for this scenario.

- The value of $\mathcal{R}_{\text {co }} / \mathcal{R}$ is constant when the value of $\mathrm{P}_{1}$ and $\mathrm{P}_{2}$ is less than 0.2 because of the low transmission rate between $S_{1}$ and $R_{1}$ or between $S_{2}$ and $\mathrm{R}_{2}$.

Fig.7c shows ratio $\mathcal{R}_{\text {co }} / \mathcal{R}$ versus $\mathrm{P}_{1}$ for cooperative network coding. The results show the following:

- The value of $\mathcal{R}_{\text {co }} / \mathcal{R}$ is always greater than one since nodes $\mathrm{S}_{2}$ and $\mathrm{R}_{1}$ can transmit packets simultaneously. For this reason, cooperative network coding is always beneficial for this scenario.

- The value of $\mathcal{R}_{\text {co }} / \mathcal{R}$ continuously increases with the increasing of $\mathrm{P}_{1}$ and $\mathrm{P}_{2}$ since nodes $\mathrm{S}_{1}, \mathrm{R}_{1}$ and nodes $\mathrm{S}_{2}$ and $\mathrm{R}_{2}$ can transmit packets at the same time.

Fig.7d shows ratio $\mathcal{R}_{\text {co }} / \mathcal{R}$ versus $\mathrm{P}_{1}$ for cooperative CSMA/CA, ideal cooperative MAC, and cooperative network coding. These results indicate the following:

- In ideal cooperative MAC, and cooperative network coding, nodes $S_{2}$ and $R_{1}$ can transmit packets at the same time. So, these protocols are always beneficial for this scenario.
- In cooperative CSMA/CA, nodes $\mathrm{R}_{1}$ and $\mathrm{S}_{2}$ cannot transmit packets at the same time. Therefore, cooperative CSMA/CA is non-beneficial in this scenario.

- The value of $\mathcal{R}_{\text {co }} / \mathcal{R}$ is constantly decreasing for cooperative CSMA and cooperative CSMA/CA when increasing the value of $\mathrm{P}_{1}$ and $\mathrm{P}_{2}$ because nodes $S_{1}, R_{1}$ and nodes $S_{2}$ and $R_{2}$ cannot transmit at the same time. While the value of $\mathcal{R}_{\text {co }} / \mathcal{R}$ is constantly decreasing for cooperative network coding since pair $S_{1}, R_{1}$ and pair $S_{2}, R_{2}$ can transmit at the same time.

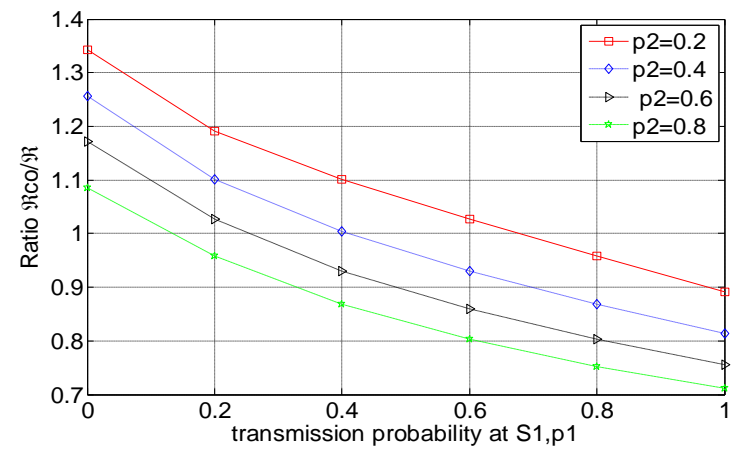

Fig. 7a. Ratio $\mathcal{R}_{\text {co }} / \mathcal{R}$ versus $\mathrm{P}_{1}$ for cooperative CSMA/CA with different value of $P_{2}$

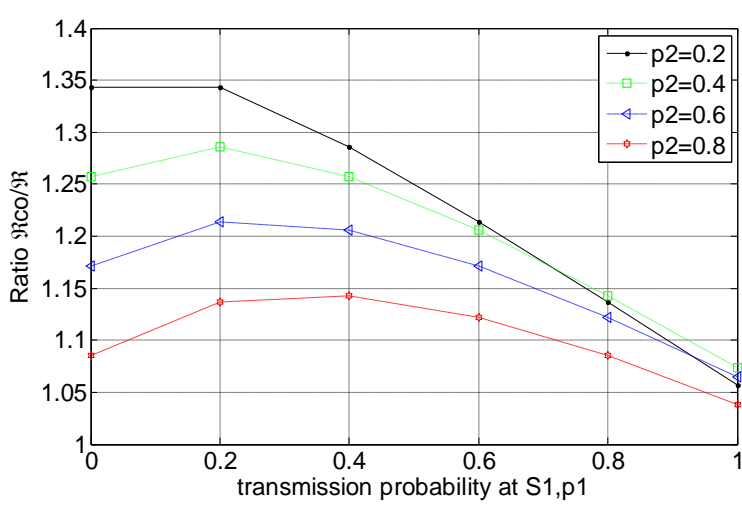

Fig. 7b. Ratio $\mathcal{R}_{\text {co }} / \mathcal{R}$ versus $P_{1}$ for ideal cooperative MAC with different value of $P_{2}$.

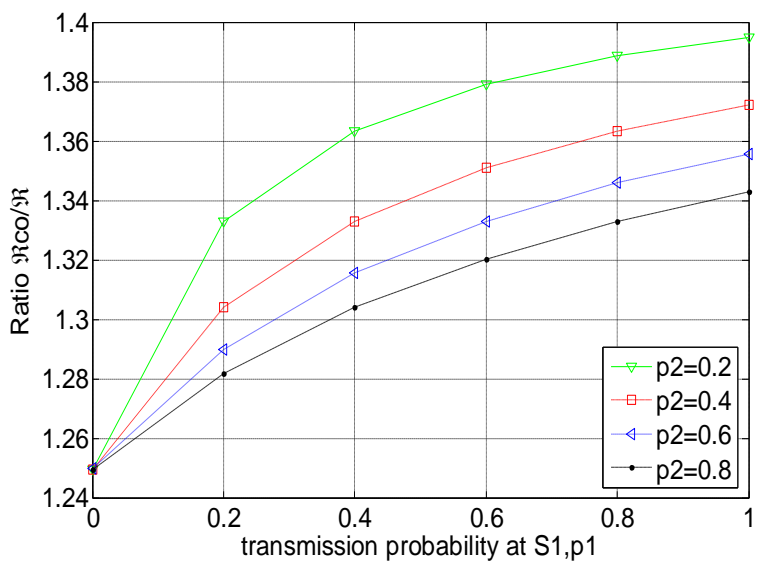

Fig. 7c. Ratio $\mathcal{R}$ co $/ \mathcal{R}$ versus $P_{1}$ for cooperative network coding with different value of $\mathbf{P}_{2}$. 


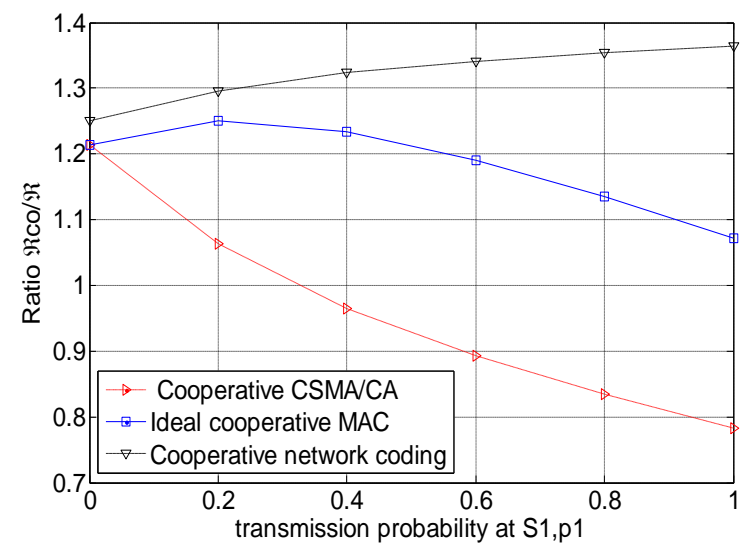

Fig. 7d. Ratio $\mathcal{R}_{\text {co }} / \mathcal{R}$ versus $\mathrm{P}_{1}$ for cooperative CSMA/CA, Ideal cooperative MAC protocol and cooperative network coding with different values of $\mathbf{P}_{\mathbf{2}}$.

The second stage, plot ratio $\mathcal{R}_{\mathrm{co}} / \mathcal{R}$ versus $\propto_{1}$ with different value of $\propto_{2}=0.2,0.4,0.6$ and 0.8 . Also, assume the fixed value of $\mathrm{P}_{1}=\mathrm{P}_{2}=0.75 ; \propto_{\mathrm{R} 1}=\propto_{\mathrm{R} 2}=0$.

Fig.8a shows ratio $\mathcal{R}_{\text {co }} / \mathcal{R}$ versus $\propto_{1}$ for cooperative CSMS/CA. The results show the following:

- The value of $\mathcal{R}_{\text {co }} / \mathcal{R}$ is less than one because $\mathrm{R}_{1}$ and $\mathrm{S}_{2}$ cannot transmit packets at the same time. For this reason, cooperative relaying in this protocol is nonbeneficial for this scenario.

- Cooperation in this protocol is only beneficial when full direct link fails to transmit packets between all pairs.

Fig.8b shows ratio $\mathcal{R}_{\text {co }} / \mathcal{R}$ versus $\propto_{1}$ for ideal cooperative MAC. These results show the following:

- The value of $\mathcal{R}_{\text {co }} / \mathcal{R}$ is one when the value of $\propto_{1}$ and $\propto_{2}$ are less than a certain threshold since the transmission through the direct link is successful.

- The value of $\mathcal{R}_{\text {co }} / \mathcal{R}$ is more than one when the values of $\propto_{1}$ and $\propto_{2}$ are more than a certain threshold because the transmission through the direct link is failed.

- The value of $\mathcal{R}_{\text {co }} / \mathcal{R}$ increases when the value of $\propto_{1}$ and $\propto_{2}$ increases since the cooperative link is only used when the error in the direct path is larger than a current threshold.

Fig.8c shows ratio $\mathcal{R}_{\text {co }} / \mathcal{R}$ versus $\propto_{1}$ for cooperative network coding. The results show the following:

- The value of $\mathcal{R}_{\text {co }} / \mathcal{R}$ is equal one when the value of $\propto_{1}$ is less than a certain threshold since the nodes only transmit via cooperative link when the value of $\propto_{1}$ is greater than a certain threshold. For this reason, the cooperation is beneficial in this protocol when values of $\propto_{1}$ and $\propto_{2}$ are greater than a certain value.

Fig.8d shows ratio $\mathcal{R}_{\text {co }} / \mathcal{R}$ versus $\propto_{1}$ for cooperative CSMA/CA, Ideal cooperative MAC and cooperative network coding. The results show the following:

- The cooperation for cooperative network coding and ideal cooperative are always beneficial in this scenario because $R_{1}$ and $S_{2}$ can transmit packets at the same time. Since the exposed terminal problem is solved for these protocols.

- The value of $\mathcal{R}_{\mathrm{co}} / \mathcal{R}$ is always less than one for cooperative CSMA/CA because of the exposed terminal problem between $\mathrm{R}_{1}$ and $\mathrm{S}_{2}$. Therefore, the cooperation in case of cooperative CSMA/CA is always non-beneficial for this scenario.

In this scenario, it is observed that, cooperation in case of cooperative CSMA/CA is non beneficial in most of the regions since $R_{1}$ and $S_{2}$ cannot transmit packets at the same time because of the exposed terminal problem between $S_{1}$ and $\mathrm{R}_{1}$. While the cooperation in case of cooperative networks coding and ideal cooperative MAC cooperative relaying is always beneficial since $R_{1}$ and $S_{2}$ can transmit packets simultaneously without affecting each other. Since an exposed terminal problem is solved for these protocols. As a conclusion, do not recommend using cooperative CSMA/CA in this scenario. In addition, we recommend using ideal cooperative MAC and cooperative network coding in this scenario.

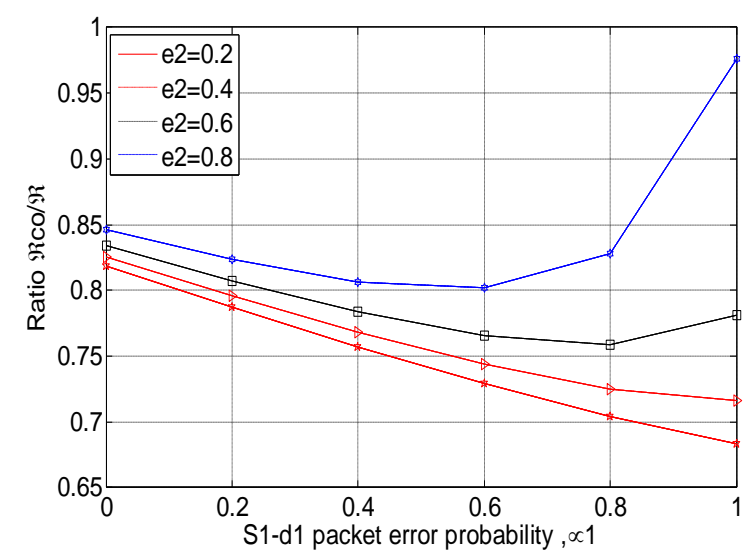

Fig. 8a. Ratio $\mathcal{R}_{\mathrm{co}} / \mathcal{R}$ versus $\propto_{1}$ for cooperative CSMA/CA with different values of $\propto_{2}$.

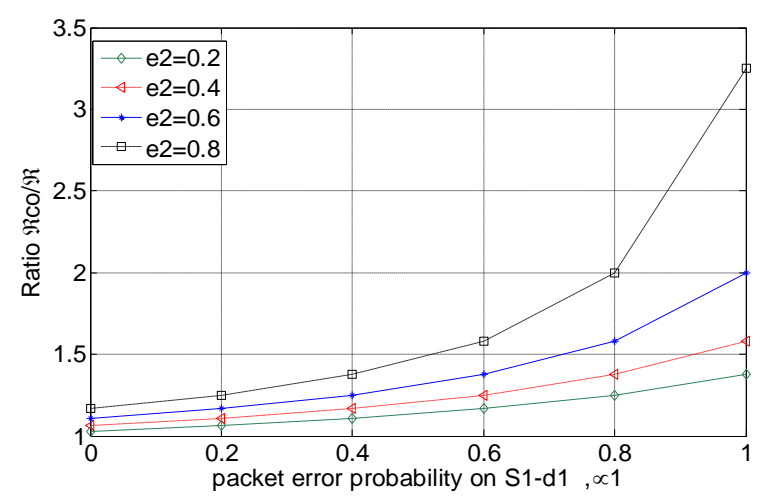

Fig. 8b. Ratio $\mathcal{R}_{\text {co }} / \mathcal{R}$ versus $\propto_{1}$ for Ideal cooperative MAC protocol with different values of $\propto_{2}$. 


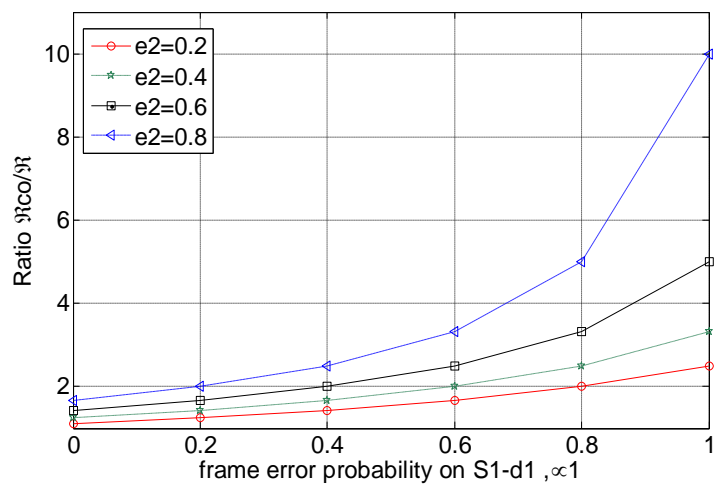

Fig. 8c. Ratio $\mathcal{R}_{\text {co }} / \mathcal{R}$ versus $\propto_{1}$ for cooperative network coding with different values of $\propto_{2}$.

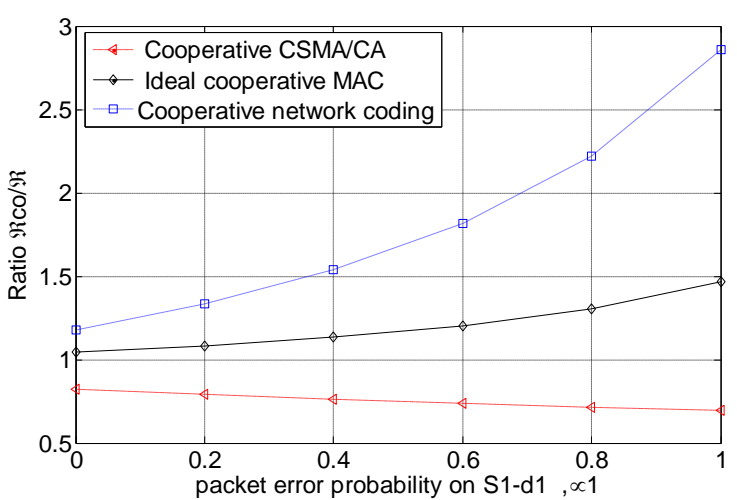

Fig. 8d. Ratio $\mathcal{R}_{\text {co }} / \mathcal{R}$ versus $\propto_{1}$ for cooperative CSMA, cooperative network coding and ideal cooperative MAC.

\subsection{Evaluation of cooperative MAC protocols for scenario two}

The cooperation for this scenario is evaluated to determine whether it is beneficial or not, for three cooperative MAC protocols by using two stages.

First stage, plot ratio $\mathcal{R}_{\mathrm{co}} / \mathcal{R}$ versus $\mathrm{P}_{1}$ with different value of $\mathrm{p}_{2}=0.2,0.4,0.6$ and 0.8 . Also, assume the fixed value of $\propto_{1}=\propto_{1}=0.3 ; \propto_{R 1}=\propto_{R 1}=0$.

Fig.9a shows ratio $\mathcal{R}_{\mathrm{co}} / \mathcal{R}$ versus $\mathrm{P}_{1}$ for cooperative CSMA protocol. These results show the following:

- The value of $\mathcal{R}_{\text {co }} / \mathcal{R}$ decreases when the value of $\mathrm{P}_{1}$ increase for all values of $\mathrm{P}_{2}$ since $\mathrm{R}$ cannot receive more than one packet at the same time.

- The value of $\mathcal{R}_{\text {co }} / \mathcal{R}$ is more than one when the values of $\mathrm{P}_{1}$ and $\mathrm{P}_{2}$ are less than one since the node $\mathrm{R}$ cannot help more than one node at the same time. For this reason, cooperative relaying for this protocol is beneficial when the values of $P_{1}$ and $P_{2}$ are less than one, but when the values of $P_{1}$ and $P_{2}$ are equal to one the cooperation and noncooperation are equal.

- The value of $\mathcal{R}_{\text {co }} / \mathcal{R}$ decreases when the values of $\mathrm{P}_{1}$ and $\mathrm{P}_{2}$ increases because of the interference between $S_{1}$ and $S_{2}$ increases.

Fig.9b shows ratio $\mathcal{R}_{\mathrm{co}} / \mathcal{R}$ versus $\mathrm{P}_{1}$ for cooperative CSMA/CA protocol. The results show the following:
- The value of $\mathcal{R}_{\mathrm{co}} / \mathcal{R}$ is less than one when the value of $\mathrm{P}_{1}$ and $\mathrm{P}_{2}$ are greater than a certain threshold because $S_{1}$ and $S_{2}$ cannot transmit packets simultaneously. For this reason, the cooperation in case of cooperative CSMA/CA is beneficial when the values of $\mathrm{P}_{1}$ and $\mathrm{P}_{2}$ are less than certain threshold.

- The value of $\mathcal{R}_{\text {co }} / \mathcal{R}$ decreases when the values of $\mathrm{P}_{2}$ and $\mathrm{P}_{1}$ increase since $\mathrm{S}_{1}$ cannot transmit pockets when $S_{2}$ is starting transmission and vice versa.

Fig.9c shows ratio $\mathcal{R}_{\text {co }} / \mathcal{R}$ versus $\mathrm{P}_{1}$ for cooperative network coding. The results show the following:

- $\quad$ The value of $\mathcal{R}_{\text {co }} / \mathcal{R}$ increases when the value of $\mathrm{P}_{1}$ and $\mathrm{P}_{2}$ increases since $\mathrm{R}$ can help more than one node at the same time.

- The value of $\mathcal{R}_{\text {co }} / \mathcal{R}$ is always greater than one since $\mathrm{S}_{1}, \mathrm{~S}_{2}$ and $\mathrm{R}$ can transmit packets at the same time. For these reasons, the cooperation for cooperative network coding is always beneficial.

- The value of $\mathcal{R}_{\mathrm{co}} / \mathcal{R}$ is constantly increasing when the value of $\mathrm{P}_{1}$ and $\mathrm{P}_{2}$ increases since the nodes $\mathrm{S}_{1}$, $\mathrm{S}_{2}$ and $\mathrm{R}$ can transmit packets at the same time.

Fig.9d shows ratio $\mathcal{R}_{\mathrm{co}} / \mathcal{R}$ versus $\mathrm{P}_{1}$ for cooperative CSMA, cooperative CSMA/CA and cooperative network coding. These results show the following:

- The performance of cooperative network coding is better than of the performance of cooperative CSMA and cooperative CSMA/CA. While the performance of cooperative CSMA/CA is better than the performance of cooperative CSMA.

- In cooperative network coding, node $\mathrm{R}$ can help more than one node at the same time since an exposed terminal problem and hidden terminal problems are solved in this protocol. Therefore, the value of $\mathcal{R}_{\text {co }} / \mathcal{R}$ always increasing.

- The value of $\mathcal{R}_{\mathrm{co}} / \mathcal{R}$ decreases when the value of $\mathrm{P}$ and $\mathrm{P}_{2}$ increases for cooperative CSMA and cooperative CSMA/CA since node $\mathrm{R}$ cannot serve more than one node at the same time.

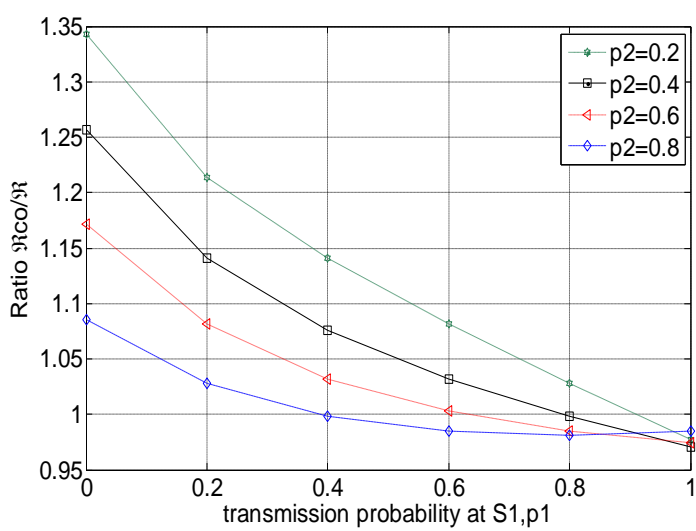

Fig. 9a. Ratio $\mathcal{R}_{\text {co }} / \mathcal{R}$ versus $\mathrm{P}_{1}$ for cooperative CSMA protocol with different values of $p_{2}$. 


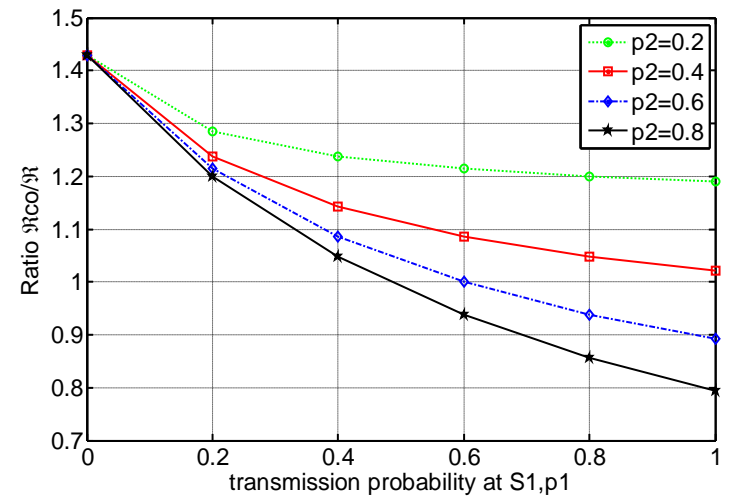

Fig. 9b. Ratio $\mathcal{R}_{\text {co }} / \mathcal{R}$ versus $P_{1}$ for cooperative CSMA/CA protocol with different values of $P_{2}$.

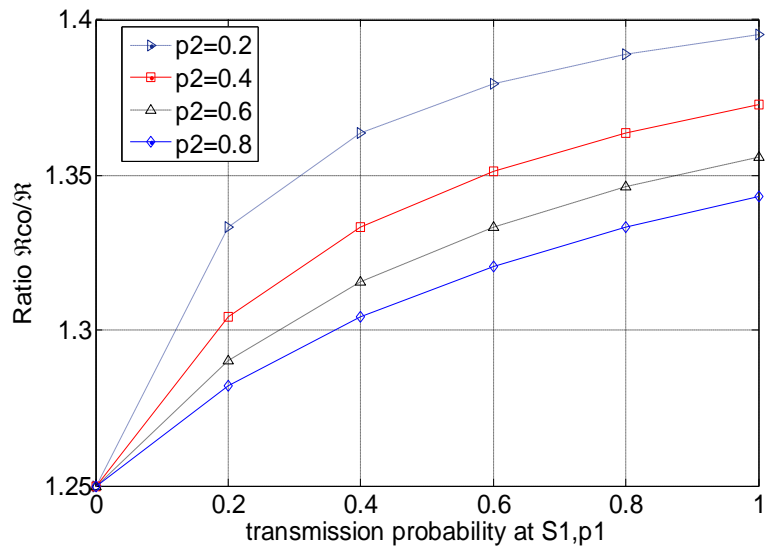

Fig. 9c. Ratio $\mathcal{R}_{\text {co }} / \mathcal{R}$ versus $P_{1}$ for cooperative network coding with different values of $P_{2}$.

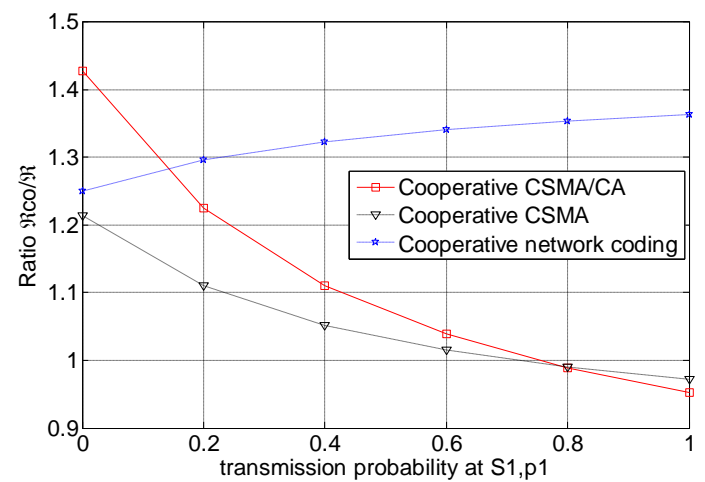

Fig. 9d. Ratio $\mathcal{R}_{\text {co }} / \mathcal{R}$ versus $p_{1}$ for cooperative CSMA/CA protocol, cooperative CSMA protocol and cooperative network coding protocol.

Second stage, plot ratio $\mathcal{R}_{\mathrm{co}} / \mathcal{R}$ versus $\propto_{1}$ with different value of $\propto_{2}=0.2,0.4,0.6$ and 0.8. Also, assume the fixed value of $\mathrm{P}_{1}=\mathrm{P}_{2}=0.75 ; \propto_{\mathrm{R} 1}=\propto_{\mathrm{R} 1}=0$

Fig.10a shows ratio $\mathcal{R}_{\mathrm{co}} / \mathcal{R}$ versus $\propto_{1}$ for cooperative CSMA. These results indicate the following:

- The value of $\mathcal{R}_{\text {co }} / \mathcal{R}$ is greater than one when the value of $\alpha_{1}$ exceed a certain value since the transmission via cooperative link is only used when the transmission failed via direct link. For this reason, cooperative CSMA is beneficial when the value of $\propto_{1}$ is greater than a certain threshold.
- The value of $\mathcal{R}_{\text {co }} / \mathcal{R}$ increases when the value of $\propto_{1}$ and $\propto_{2}$ increases because the cooperative link is only used when the transmission via the direct link fails.

Fig.10 b shows ratio $\mathcal{R}_{\mathrm{co}} / \mathcal{R}$ versus $\propto_{1}$ for cooperative CSMA/CA. These results indicate the following:

- The value of $\mathcal{R}_{\text {co }} / \mathcal{R}$ is greater than one when the value of $\propto_{1}$ extends a certain threshold since $R$ retransmits packets only when transmission through the direct link fails. Therefore, cooperative CSMA/CA is beneficial when the value of $\propto_{1}$ is greater than a certain threshold.

- $\quad$ The value of $\mathcal{R}_{\text {co }} / \mathcal{R}$ increases when the value of $\propto_{1}$ and $\propto_{2}$ increases since cooperative link is used when the transmission failed through the direct link.

Fig.11c shows the ratio $\mathcal{R}_{\mathrm{co}} / \mathcal{R}$ versus $\propto_{1}$ for cooperative network coding. These results indicates the following:

- The cooperation in this scenario is beneficial when the value of $\propto_{1}$ exceeds a certain threshold.

- The value of $\mathcal{R}_{\text {co }} / \mathcal{R}$ increases when the value of $\propto$ and $\propto_{2}$ increases since the cooperative link is only forward packets when the transmission via direct link fails.

Fig.10d shows ratio $\mathcal{R}_{\mathrm{co}} / \mathcal{R}$ versus $\propto_{1}$ for cooperative CSMA, cooperative CSAM/CA, and cooperative network coding. These results indicate the following:

- The cooperation for all studied cooperative MAC protocols is beneficial when the value of $\propto_{1}$ exceed a certain value. The reason for that, the cooperative link is only used when the direct link of the transmitter is failed.

- The performance of cooperation network coding is better than the performance of cooperative CSMA protocol and cooperative CSMA/CA protocol.

In this scenario, the cooperation for cooperative CSMA and cooperative CSMA/CA is beneficial in the most regions. While the cooperation for cooperative network coding is always beneficial. Therefore, the cooperative CSMA and cooperative CSMA/CA is recommended when the values of $\propto$ and $p$ are less than a certain value using for this scenario. On the other hand, using cooperative network coding is always recommended for this scenario.

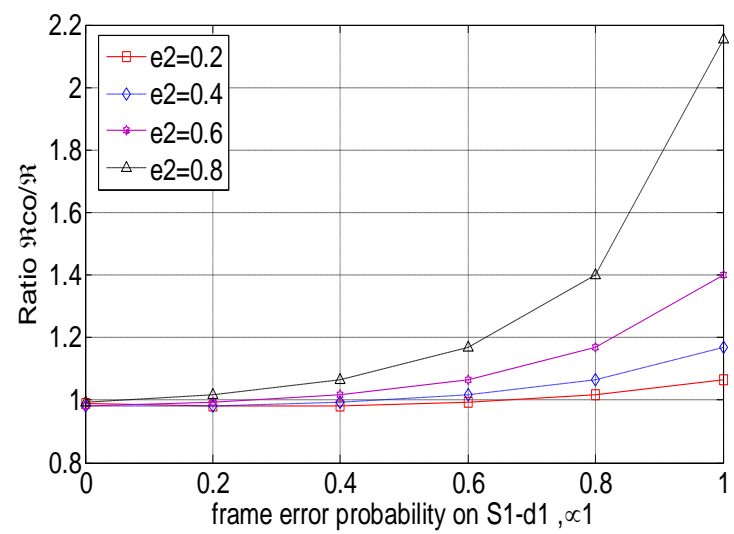

Fig.10a. Ratio $\mathcal{R}_{\mathrm{co}} / \mathcal{R}$ versus $\propto_{1}$ for cooperative CSMA with different value of $\propto_{2}$. 


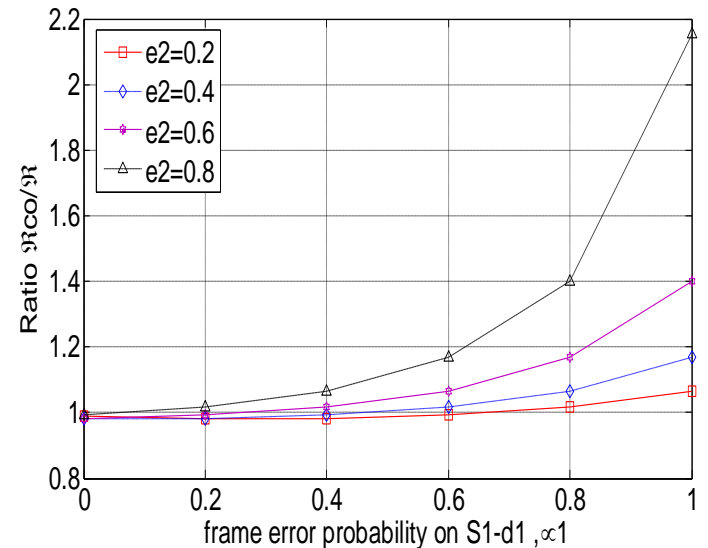

Fig.10b. Ratio $\mathcal{R}_{\text {co }} / \mathcal{R}$ versus $\propto_{1}$ for Cooperative CSMA/CA with different value of $\propto_{2}$.

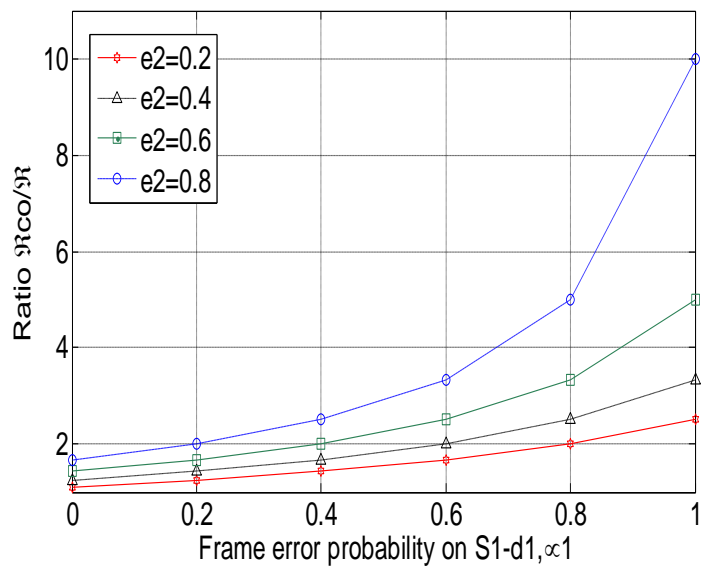

Fig. 10c. Ratio $\mathcal{R}_{\text {co }} / \mathcal{R}$ versus $\propto_{1}$ for cooperative network coding protocol with different value of $\propto_{2}$.

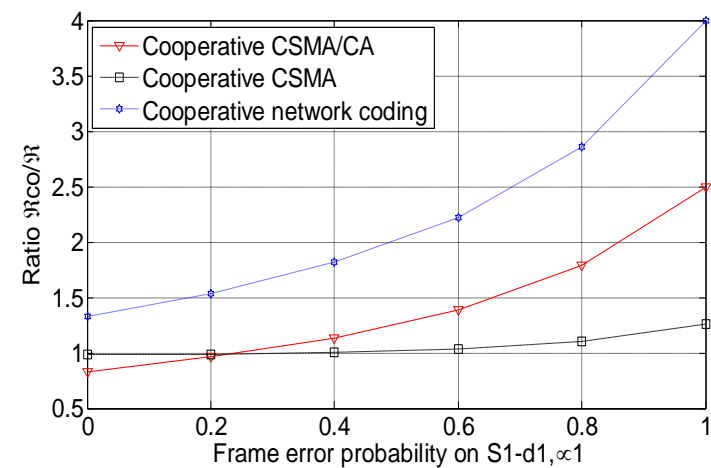

Fig. 10d. Ratio $\mathcal{R}_{\mathrm{co}} / \mathcal{R}$ versus $\propto_{1}$ for cooperative CSMA, cooperative CSMA and cooperative network coding.

\subsection{Evaluation of cooperative MAC protocols for scenario three}

First stage, we plot ratio $\mathcal{R}_{\mathrm{co}} / \mathcal{R}$ versus $\mathrm{P}_{1}$ with different value of $\mathrm{p}_{2}=0.2,0.4,0.6$ and 0.8 . Also, assume the fixed value of $\propto_{1}=\propto_{2}=\propto_{3}=0.3 ; \propto_{R_{1}}=\propto_{R_{2}}=0 ; P_{2}=0.75$.

Fig.11a shows ratio $\mathcal{R}_{\mathrm{co}} / \mathcal{R}$ versus $\mathrm{P}_{1}$ for cooperative CSMA. The results show the following:

- The value of $\mathcal{R}_{\text {co }} / \mathcal{R}$ is close to one because $\mathrm{R}$ cannot receive more than one packet at the same time.
Therefore, the cooperation and non-cooperation for this protocol are equal.

Fig.11b shows ratio $\mathcal{R}_{\text {co }} / \mathcal{R}$ versus $\mathrm{P}_{1}$ for cooperative CSMA/CA. These results indicate the following:

- The value of $\mathcal{R}_{\mathbf{c o}} / \mathcal{R}$ is always less than one since as node $S_{1}$ or $S_{2}$ or $S_{3}$ start transmitting packets other nodes cannot transmit at the same time. For this reason, the cooperation in this protocol is always non-beneficial in this scenario.

Fig.11c shows ratio $\mathcal{R}_{\mathrm{co}} / \mathcal{R}$ versus $\mathrm{P}_{1}$ for cooperative network coding protocol. These results indicate the following:

- The value of $\mathcal{R}_{\text {co }} / \mathcal{R}$ is always greater than one because all nodes can transmit packets at the same time. For this reason, the cooperation in this protocol is beneficial.

Fig.11d shows ratio $\mathcal{R}_{\text {co }} / \mathcal{R}$ versus $\mathrm{P}_{1}$ for cooperative CSMA protocol, cooperative CSMA/CA protocol and cooperative network coding protocol. These results show the following:

- The Cooperation in cooperative network coding protocol is beneficial since all nodes can transmit packets simultaneously. While the cooperation in cooperative CSMA protocol and cooperative CSMA/CA protocol is non-beneficial since only one node can transmit packets at the same time.

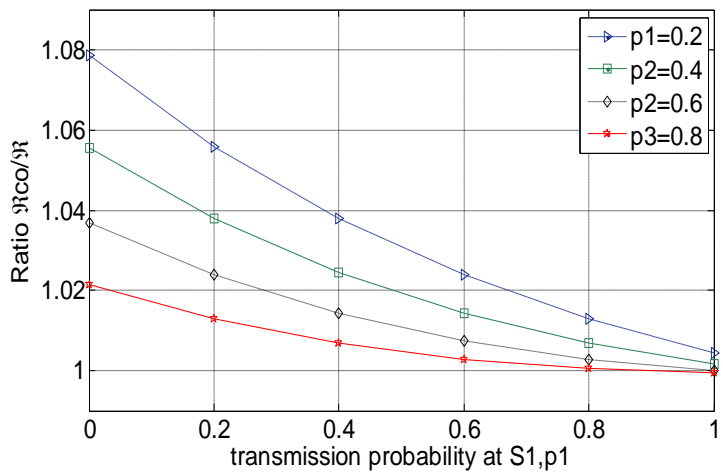

Fig. 11a. Ratio $\mathcal{R}_{\text {co }} / \mathcal{R}$ versus $P_{1}$ for cooperative CSMA protocol with different values of $p_{2}$.

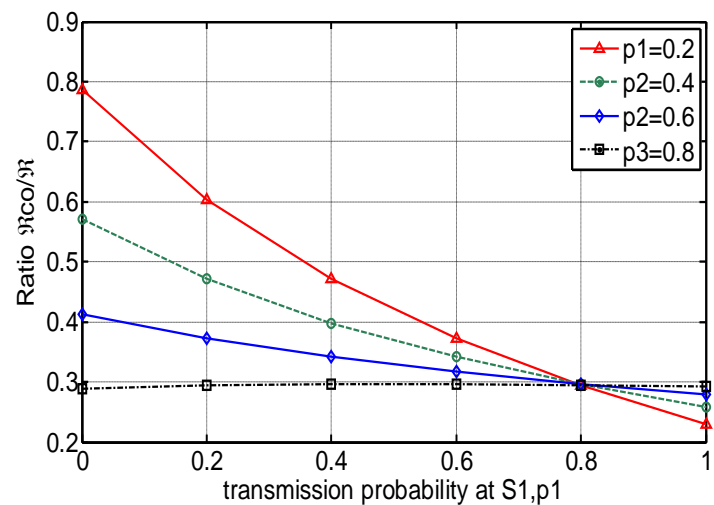

Fig. 11b. Ratio $\mathcal{R}_{\text {co }} / \mathcal{R}$ versus $P_{1}$ for Cooperative CSMA/CA protocol with different values of $P_{2}$. 


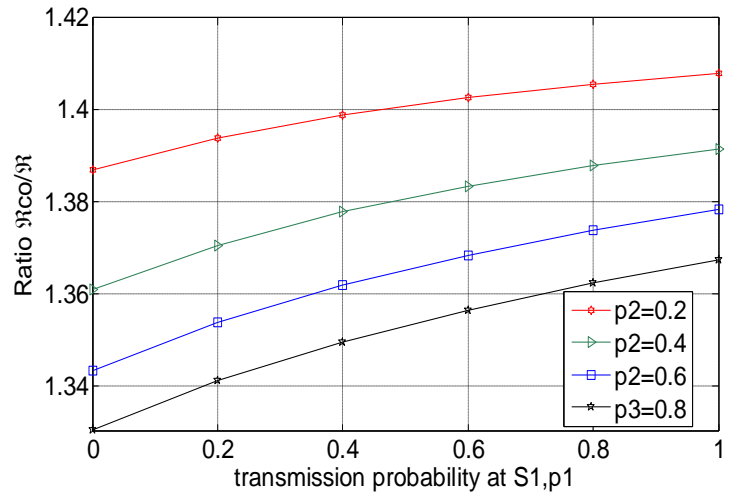

Fig.11c. Ratio $\mathcal{R}_{\text {co }} / \mathcal{R}$ versus $P_{1}$ for cooperative network coding protocol with different values of $\mathbf{P}_{2}$.
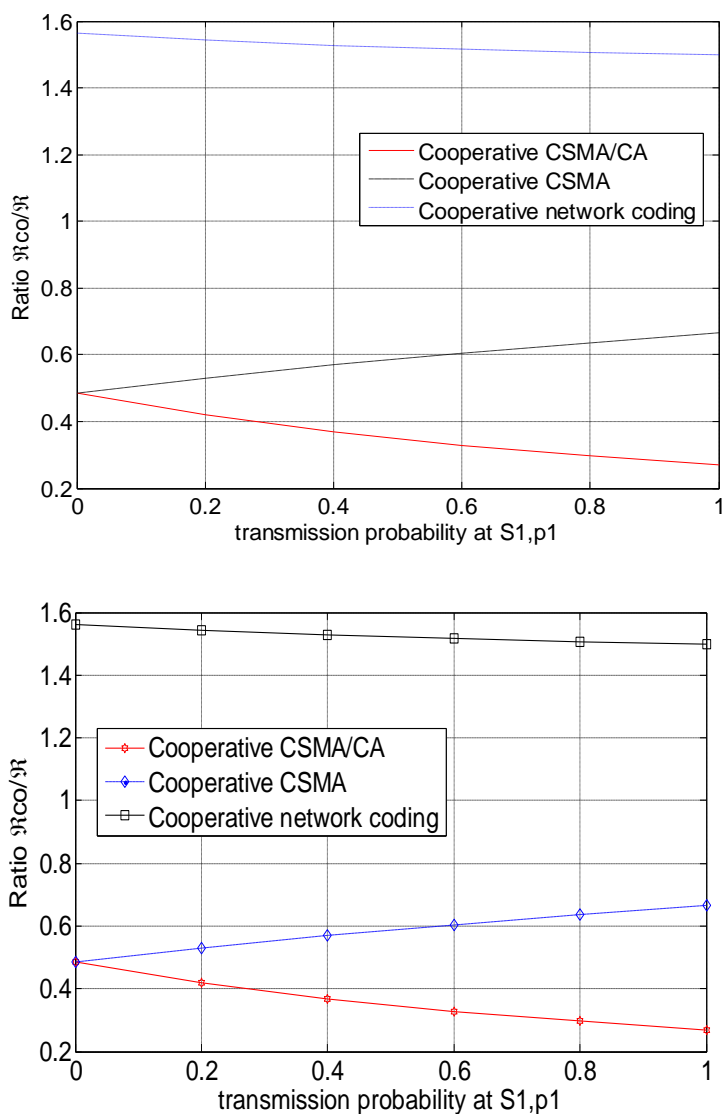

Fig.11d. Ratio $\mathcal{R}_{\text {co }} / \mathcal{R}$ versus $\propto_{1}$ for cooperative CSMA protocol, cooperative CSMA protocol and cooperative network coding protocol.

Second stage, we plot ratio $\mathcal{R}_{\mathrm{co}} / \mathcal{R}$ versus $\propto_{1}$ with different value of $\propto_{1}=0.2,0.4,0.6$ and 0.8 . Also, assume the fixed value of $P_{1}=P_{2}=P_{3}=0.75 ; \propto_{R_{1}}=\propto_{R_{2}}=\propto_{R_{3}}=\propto_{R_{4}}=0 ; \propto_{3}=0.3$.

Fig.12a shows ratio $\mathcal{R}_{\mathrm{co}} / \mathcal{R}$ versus $\propto_{1}$ for cooperative CSMA. These results show the following

- The value of $\mathcal{R}_{\text {co }} / \mathcal{R}$ is closed to one because the node $\mathrm{R}$ cannot help more than one node at the same time. For this reason, the cooperation and noncooperation is equivalent in this case.

Fig.12b shows ratio $\mathcal{R}_{\mathrm{co}} / \mathcal{R}$ versus $\propto_{1}$ for cooperative CSMA/CA. These results show the following
- The value of $\mathcal{R}_{\text {co }} / \mathcal{R}$ is always less than one, where the overall throughput is decreased to more than half because only one node can transmits packets at the same time.

- The cooperation in this protocols is always non beneficial since only one node can transmit packets at the same time.

Fig.12c shows ratio $\mathcal{R}_{\text {co }} / \mathcal{R}$ versus $\propto_{1}$ for cooperative network coding. These results indicate the following

- The value of $\mathcal{R}_{\mathrm{co}} / \mathcal{R}$ in this protocol is more than one since only one node can transmit packets at the same time because the interference and collision are solved for this protocol. For this reason, the cooperation for this protocol is always beneficial.

- The value of $\mathcal{R}_{\text {co }} / \mathcal{R}$ increasing when the values of $\propto_{1}$ and $\propto_{2}$ increasing due to the transmission via cooperative link is only when the transmission by direct link fails.

Fig. $12 \mathrm{~d}$ shows ratio $\mathcal{R}_{\mathrm{co}} / \mathcal{R}$ versus $\boldsymbol{\propto}_{1}$ for cooperative CSMA protocol, cooperative CSMA/CA protocol and cooperative network coding protocol. These results show the following

- The value of $\mathcal{R}_{\text {co }} / \mathcal{R}$ is greater than one for cooperative network coding, while it is equal one for cooperative CSMA, but it is less than one for cooperative CSMA/CA.

- In this scenario, the cooperation is non-beneficial for cooperative CSMA/CA, while it is beneficial for cooperative network coding and cooperative CSMA.

For this scenario, it can be observed that, the cooperation when using cooperative CSMA and cooperative CSMA/CA are always non-beneficial. While the cooperation in case of cooperative network coding is always beneficial. Therefore, we do not recommend using cooperative CSMA and cooperative CSMA/CA in this scenario. On the other hand, cooperative network coding is always recommended in this scenario

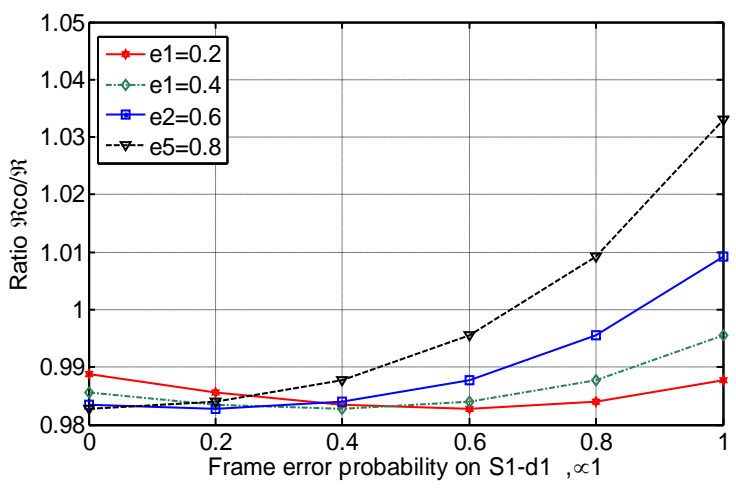

Fig.12a. Ratio $\mathcal{R}_{\text {co }} / \mathcal{R}$ versus $\propto_{1}$ for cooperative CSMA protocol with different value of $\propto_{2}$. 


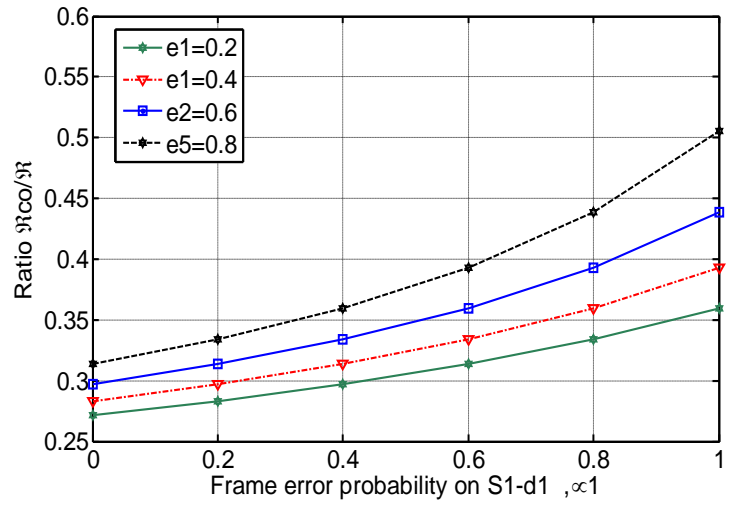

Fig.12b. Ratio $\mathcal{R}_{\text {co }} / \mathcal{R}$ versus $\propto_{1}$ for cooperative CSMA/CA with different value of $\propto_{2}$.

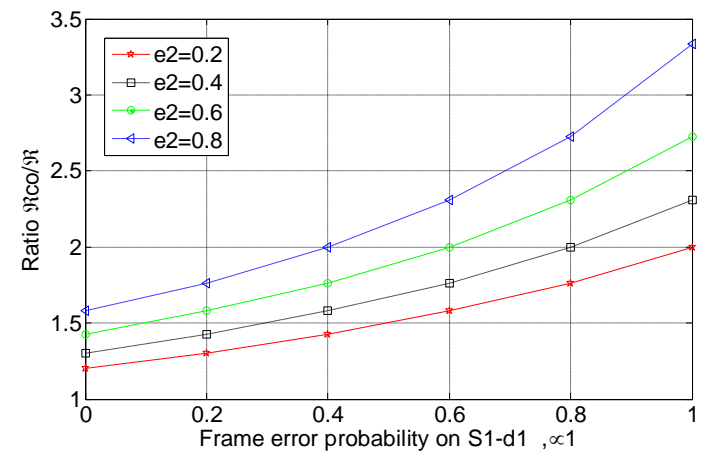

Fig.12c. Ratio $\mathcal{R}_{\text {co }} / \mathcal{R}$ versus $\propto_{1}$ for cooperative network coding with different values of $\propto_{2}$.

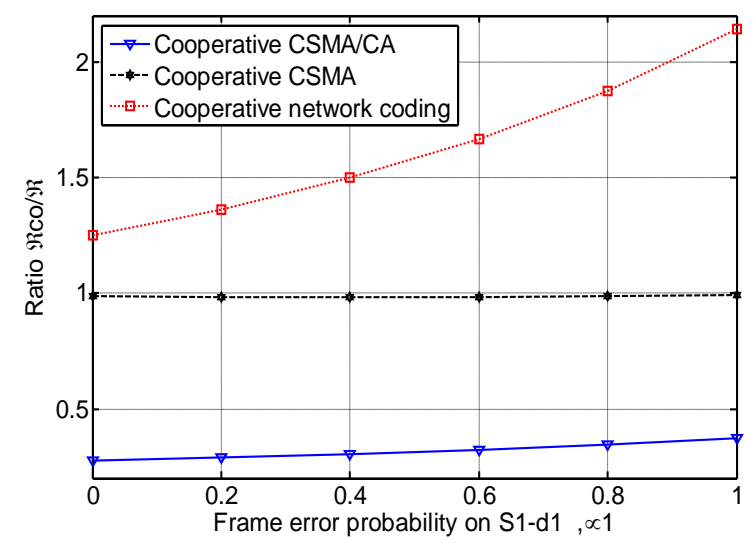

Fig. 12d. Ratio $\mathcal{R}_{\text {co }} / \mathcal{R}$ versus $\propto_{1}$ for cooperative CSMA, cooperative CSMA and cooperative network coding.

\subsection{Evaluation of cooperative MAC protocols for scenario four}

In this scenario, the overall throughput in cooperative CSAM, cooperative CSMA/CA, and ideal cooperative MAC are equal.

First stage, plot ratio $\mathcal{R}_{\mathrm{co}} / \mathcal{R}$ versus $\mathrm{p}_{1}$ with different the value of $\mathrm{P}_{2}=0.2,0.4,0.6$ and 0.8. Also, assume fixed the value of $\propto_{1}$ $=\propto_{2}=\propto_{3}=\propto_{4}=0.3 ; \propto_{R_{1}}=\propto_{R_{2}}=\propto_{R_{3}}=\propto_{R_{4}}=0 ; P_{3}=P_{4}=0.75$.

Fig.13a shows ratio $\mathcal{R}_{\mathrm{co}} / \mathcal{R}$ versus $\mathrm{p}_{1}$ in contention MAC protocol. The results show the following:
- The value of $\mathcal{R}_{\text {co }} / \mathcal{R}$ is always greater than one because each relay help only one pair. For this reason, cooperative relaying in this scenario is beneficial.

- In this scenario, there is no interference because each pair is out of range for the other nodes.

Fig.13b shows ratio $\mathcal{R}_{\mathrm{co}} / \mathcal{R}$ versus $\mathrm{P}_{1}$ for cooperative network coding protocol. The results show the following:

- The value of $\mathcal{R}_{\mathrm{co}} / \mathcal{R}$ is always greater than one since all nodes can transmit packets at the same time without effecting each other. For this reason, cooperative relaying for this protocol is always beneficial.

- This protocol solves the interference problem since one relay can help more than one node at the same time.

Fig.13c shows ratio $\mathcal{R}_{\mathrm{co}} / \mathcal{R}$ versus $\mathrm{P}_{1}$ for cooperation in contention MAC protocol and cooperative network coding. These results indicate the following:

- The value of $\mathcal{R}_{\mathbf{c o}} / \mathcal{R}$ is always greater than one due to the interference problem for these protocols is solved.

- The overall throughput in cooperative network coding is better than the overall throughput in cooperation in contention MAC protocol that are studied in this scenario since the relay and sources in case cooperative network coding can transmit packets at the same time.

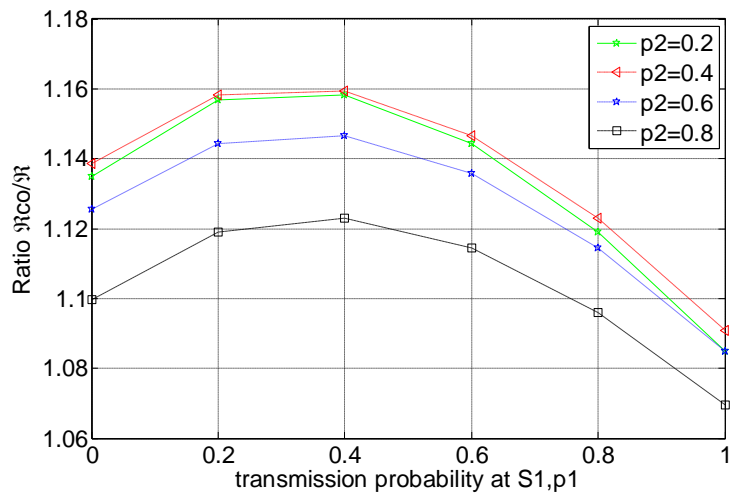

Fig.13a. Ratio $\mathcal{R}_{\mathrm{co}} / \mathcal{R}$ versus $\mathrm{P} 1$ for cooperative in contention MAC protocols with different values of $P 2$.

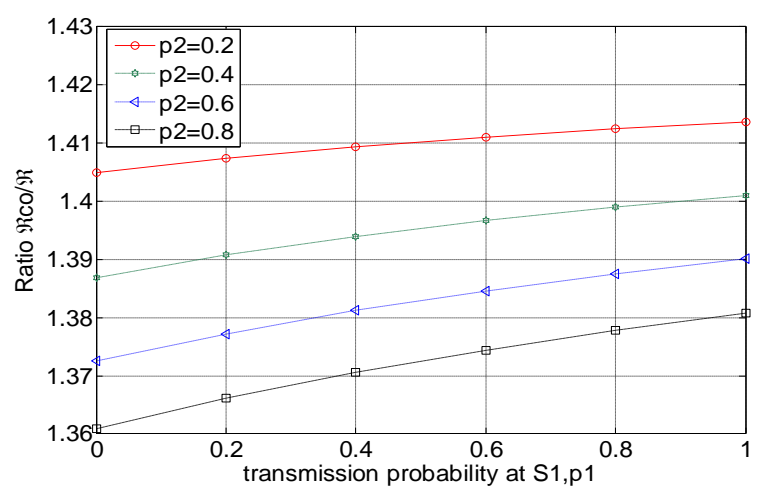

Fig.13b. Ratio $\mathcal{R}_{\text {co }} / \mathcal{R}$ versus $\mathrm{P}_{1}$ for cooperative network coding with different values of $P_{2}$. 


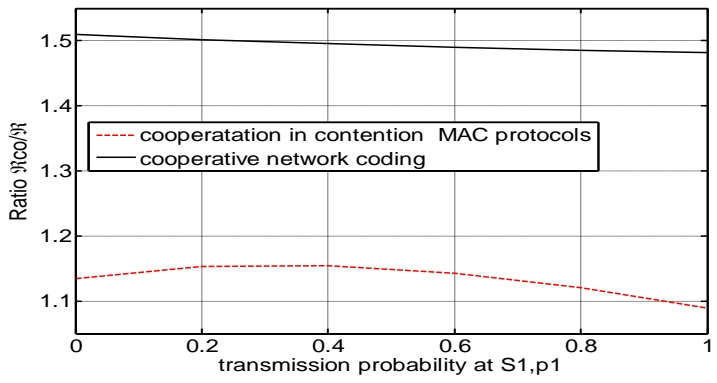

Fig. 13c. Ratio $\mathcal{R}_{\text {co }} / \mathcal{R}$ versus $P_{1}$ for cooperative network coding and cooperation in contention MAC protocol.

Second stage, we plot ratio $\mathcal{R}_{\mathrm{co}} / \mathcal{R}$ versus transmission probability $\propto_{1}$, for different values of $\propto_{2}=0.2,0.4,0.6$ and 0.8. Assume the fixed values of $\propto_{3}=\propto_{4}=0.3$; $\mathrm{P}_{1}=\mathrm{P}_{2}=\mathrm{P}_{3}=\mathrm{P}_{4}=0.75 ; \propto_{\mathrm{R}_{1}}=\propto_{\mathrm{R}_{2}}=\propto_{\mathrm{R}_{3}}=\propto_{\mathrm{R}_{4}}=0$.

Fig.14a shows ratio $\mathcal{R}_{\mathrm{co}} / \mathcal{R}$ versus $\propto_{1}$ for cooperation in contention MAC protocol. These results indicate the following:

- The value of $\mathcal{R}_{\text {co }} / \mathcal{R}$ is always greater than one because each pair is in the transmitting range of out of others. For this reason, cooperative relaying in these protocols are always beneficial.

- The value of $\mathcal{R}_{\text {co }} / \mathcal{R}$ increases continuously when the values of $\propto_{1}$ and $\propto_{2}$ increase.

Fig. $14 \mathrm{~b}$ shows ratio $\mathcal{R}_{\mathrm{co}} / \mathcal{R}$ versus $\propto_{1}$ for cooperative network coding protocol. The results show the following:

- The value of $\mathcal{R}_{\text {co }} / \mathcal{R}$ is always greater than one because all nodes can transmit packets at the same time. Therefore, the cooperation in this protocol is always beneficial.

Fig.14c shows ratio $\mathcal{R}_{\mathrm{co}} / \mathcal{R}$ versus $\propto_{1}$ for cooperative network coding protocol and cooperation in the contention MAC protocol. These results indicate the following:

- Cooperative relaying for all cooperative protocols that are studied here is always beneficial.

- The overall throughput in cooperative network coding is greater than the overall throughput in cooperation in contention MAC protocol.

In this scenario, it can be observed that, all studied cooperative MAC protocols in this scenario are beneficial. In addition, the cooperative network coding protocol is better than the other protocols.

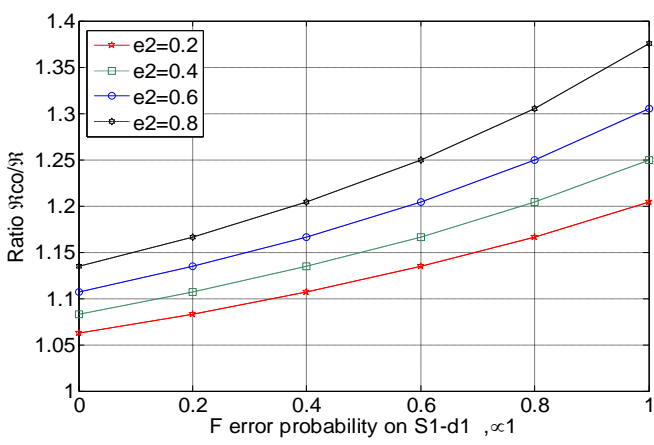

Fig. 14a. Ratio $\mathcal{R}_{\text {co }} / \mathcal{R}$ versus $\propto_{1}$ for cooperative in contention MAC protocols with different values of $\propto_{2}$.

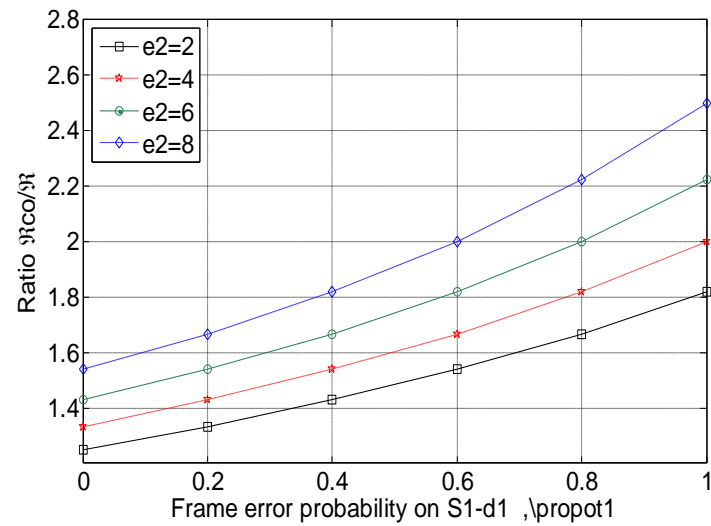

Fig. 14b. Ratio $\mathcal{R}_{\mathrm{co}} / \mathcal{R}$ versus $\propto_{1}$ for cooperative network coding with different value of $\alpha_{2}$.

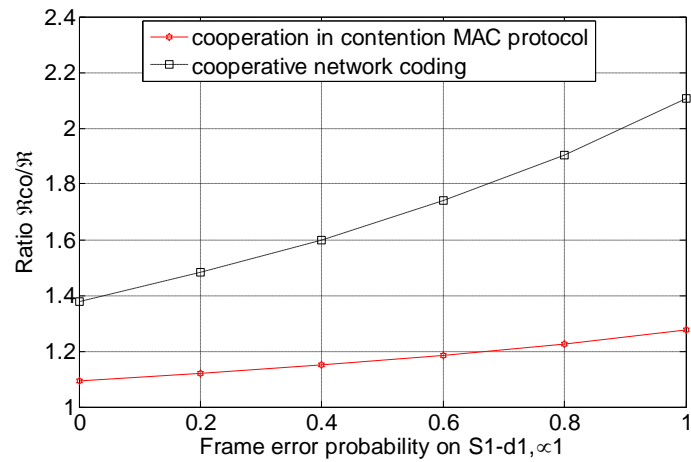

Fig. 14c. Ratio $\mathcal{R}_{\mathrm{co}} / \mathcal{R}$ versus $\propto_{1}$ for cooperative network coding and cooperation in contention MAC protocol.

\section{CONCLUSION}

In this paper, we proposed four different scenarios to evaluate the overall throughput, with and without cooperation, for four cooperative MAC protocols. We drove the mathematical model for four cooperative protocols (cooperative CSMA, cooperative CSMA/CA, ideal cooperative MAC and cooperative network coding) in the proposed scenarios. In addition, the overall throughput is evaluated to determine whether these protocols are beneficial or not in the proposed four different scenarios.

The results showed that the overall throughput in networks with cooperative relaying for cooperative CSMA, cooperative CSMA/CA and ideal cooperative MAC is sharply decreased when the couples that using one relay is increased. That is the situation in scenario two and three. For this reason, it is recommended for these cooperation protocols to have one relay for each pair as it is clear in scenario four.

In addition, the overall throughput in the networks with cooperative relaying is not affected as the number of nodes that is using the same relay increased for all proposed scenarios. Moreover, cooperative relaying protocol outstood the other protocols in the proposed scenarios. Unfortunately, although cooperative relaying protocol is always beneficial in all scenarios, it increases the complexity and overhead in the network. 


\section{REFERENCES}

[1] J. Laneman, D. Tse, and G. Wornell, "Cooperative Diversity in Wireless Networks: Efficient Protocols and Outage Behavior," IEEE Trans. Inf. Theory, vol. 50, no. 12, pp. 3062-3080, 2004.

[2] P. Ju, W. Song, and D. Zhou, "Survey on cooperative medium access control protocols," Iet Commun., vol. 7, no. 9, pp. 893-902, 2013.

[3] Z. Haas and T. Chen, "Cluster-based cooperative communication with network coding in wireless networks," 2010 - Milcom 2010 Mil. Commun. Conf., pp. 2082-2089, 2010.

[4] H. Adam, W. Elmenreich, C. Bettstetter, and S. M. Senouci, "CoRe-MAC: A MAC-Protocol for Cooperative Relaying in Wireless Networks," GLOBECOM 2009 - 2009 IEEE Glob. Telecommun. Conf., pp. 1-6, 2009.

[5] B. Hagelstein, M. Abolhasan, D. Franklin, F. Safaei, and W. Ni, "Analytic Performance Model for State-Based MAC Layer Cooperative Retransmission Protocols," IEEE Trans. Mob. Comput., vol. 15, no. 1, pp. 32-44, 2016.

[6] A. Bletsas, A. Bletsas, A. Khisti, A. Khisti, D. Reed, A. Lippman, and A. Lippman, "A simple Cooperative diversity method based on network path selection," Sel. Areas Commun. IEEE J., vol. 24, no. 3, pp. 659-672, 2006.

[7] G. Kramer, M. Gastpar, and P. Gupta, "Cooperative strategies and capacity theorems for relay networks," IEEE Trans. Inf. Theory, vol. 51, no. 9, pp. 3037-3063, 2005.

[8] T. E. Hunter and A. Nosratinia, "Diversity through coded cooperation," IEEE Trans. Wirel. Commun., vol. 5, no. 2, pp. 283-289, 2006.
[9] V. Kapadia, S. Patel, and R. Jhaveri, "Comparative Study of Hidden Node Problem and Solution Using Different Techniques and Protocols," J. Comput., vol. 2, no. 3, pp. 65-67, 2010.

[10] R. Ahlswede, N. Cai, S. Y. R. Li, and R. W. Yeung, "Network information flow," IEEE Trans. Inf. theory, vol. 46, no. 4, pp. 1204-1216, 2000.

[11] A. Munari, F. Rossetto, and M. Zorzi, "Phoenix: Making cooperation more efficient through network coding in wireless networks," IEEE Trans. Wirel. Commun., vol. 8, no. 10 , pp. 5248-5258, 2009.

[12] C. Chou, J. Yang, and D. Wang, "Cooperative MAC Protocol with Automatic Relay Selection in Distributed Wireless Networks," Fifth Annu. IEEE Int. Conf. Pervasive Comput. Commun. Work. , pp. 526-531, 2007.

[13] H. Shan, P. Wang, W. Zhuang, and Z. Wang, "CrossLayer Cooperative Triple Busy Tone Multiple Access for Wireless Networks," Glob. Telecommun. Conf. 2008. IEEE GLOBECOM 2008. IEEE, pp. 1-5, 2008.

[14] N. Fahmy and T. Todd, "A Selective CSMA Protocol with Cooperative Nulling for Ad Hoc Networks with Smart Antennas," pp. 387-392, 2004.

[15] Q. Liu, G. Hu, X. Wang, and X. Zhou, "A Cooperative CSMA / CA MAC for Channel-Hopping Rendezvous based Cognitive Radio Networks," pp. 539-544, 2014.

[16] N. Marchenko, "Resource-Efficient Relay Selection in Cooperative Wireless Networks," no. August 2013, 2013.

[17] N. Marchenko, C. Bettstetter, E. Yanmaz, S. Group, E. Systems, and L. Gmbh, "On Radio Resource Allocation in Proactive Cooperative Relaying," IEEE Int. Conf. Commun. Work. , pp. 1--5, 2009. 